\title{
Comparative genome analysis of Prevotella intermedia strain isolated from infected root canal reveals features related to pathogenicity and adaptation
}

Yunfeng Ruan ${ }^{1,2+}$, Lu Shen ${ }^{1,2+}$, Yan Zou ${ }^{5}$, Zhengnan Qi ${ }^{5}$, Jun Yin ${ }^{5}$, Jie Jiang ${ }^{1}$, Liang Guo ${ }^{3}$, Lin He $e^{1,2}$, Zijiang Chen ${ }^{4,1}$, Zisheng Tang ${ }^{5^{*}}$ and Shengying Qin ${ }^{1,2^{*}}$

\begin{abstract}
Background: Many species of the genus Prevotella are pathogens that cause oral diseases. Prevotella intermedia is known to cause various oral disorders e.g. periodontal disease, periapical periodontitis and noma as well as colonize in the respiratory tract and be associated with cystic fibrosis and chronic bronchitis. It is of clinical significance to identify the main drive of its various adaptation and pathogenicity. In order to explore the intra-species genetic differences among strains of Prevotella intermedia of different niches, we isolated a strain Prevotella intermedia ZT from the infected root canal of a Chinese patient with periapical periodontitis and gained a draft genome sequence. We annotated the genome and compared it with the genomes of other taxa in the genus Prevotella.

Results: The raw data set, consisting of approximately $65 \mathrm{X}$-coverage reads, was trimmed and assembled into contigs from which 2165 ORFs were predicted. The comparison of the Prevotella intermedia ZT genome sequence with the published genome sequence of Prevotella intermedia 17 and Prevotella intermedia ATCC25611 revealed that $\sim 14 \%$ of the genes were strain-specific. The Preveotella intermedia strains share a set of conserved genes contributing to its adaptation and pathogenic and possess strain-specific genes especially those involved in adhesion and secreting bacteriocin. The Prevotella intermedia ZT shares similar gene content with other taxa of genus Prevotella. The genomes of the genus Prevotella is highly dynamic with relative conserved parts: on average, about half of the genes in one Prevotella genome were not included in another genome of the different Prevotella species. The degree of conservation varied with different pathways: the ability of amino acid biosynthesis varied greatly with species but the pathway of cell wall components biosynthesis were nearly constant. Phylogenetic tree shows that the taxa from different niches are scarcely distributed among clades.
\end{abstract}

Conclusions: Prevotella intermedia ZT belongs to a genus marked with highly dynamic genomes. The specific genes of Prevotella intermedia indicate that adhesion, competing with surrounding microbes and horizontal gene transfer are the main drive of the evolution of Prevotella intermedia.

\footnotetext{
* Correspondence: tangzisheng163@163.com; chinsir@sjtu.edu.cn

${ }^{\dagger}$ Equal contributors

${ }^{5}$ Department of Endodontics, 9th People's Hospital, Shanghai JiaoTong University, School of Medicine, Shanghai Key Laboratory of Stomatology, Shanghai 200011, China

'Bio-X Institutes, Key Laboratory for the Genetics of Developmental and Neuropsychiatric Disorders(Ministry of Education, Shanghai Jiao Tong University, 1954 Huashang Road, Shanghai 200030, China

Full list of author information is available at the end of the article
} 


\section{Background}

Prevotella, previously classified in the genus Bacteroides, was a genus of an obligate anaerobic gram-negative rodshape bacterium. They are classified as "black pigmented bacteria" as they form shiny and smooth colonies with grey, light brown or black color on blood agar plate [1]. Although they generally have a limited ability to ferment amino acid and require hemin and menadione to grow, Prevotella is a versatile genus which has been observed in various niches, such as oral cavity, upper respiratory tract, urogenital tract [2], rumen and human feces [3]. Many Prevotella taxa from oral cavity are potential/opportunistic pathogens. Gomes et al have shown that Prevotella spp is associated with pain and swelling and 'wet' canal of oral diseases [4-6]. Besides, Prevotella intermdedia, Prevotella dentalis, Prevotella denticola, Prevotella meleninogenica are known to be pathogenic under a wide range of situations and invade into the host tissues [7-9].

Prevotella intermediahas long been known to be associated with periodontal diseases [10-12], periapical periodontitis $[4,5,13]$ and noma (an acute gangrenous disease) $[14,15]$. Prevotella intermedia also exists at the diseased site of periapical periodontitis $[13,16,17]$ and shows a significantly higher detection ratio in symptomatic sites than in asymptomatic sites [13]. Besides involved in oral diseases, Prevotella intermedia was also reported to colonize in the respiratory tract and be associated with cystic fibrosis and chronic bronchitis $[6,18]$. The association of Prevotella intermedia with various diseases raises a question what drives the evolution of Prevotella intermedia in different niches. The question also helps us predict how much gene mutation will take for a strain adapt to another niche.

There have been evidences to show that the intra-species difference exists among Prevotella intermedia strains in different niches, e.g. the degradative enzyme activity of Prevotella intermedia in diseased sites is significantly higher than in healthy sites [19]. However, the difference of the phenotype directly related with pathogenicity could be either genetic or non-genetic such as the regulation of gene expression under different niches and interaction with surrounding microbe. Previous study proved that the intra-species differences of some genes caused different bacterial adaptation to the environment $[20,21]$. The intra-species variation could be an essential factor to these differences [22]. Therefore, our approach to solve this issue is to assume the drive is genetic factors and explore the intra-species variation among different strains of Prevotella intermedia.

The first published genome sequence of Prevotella intermedia is that of Prevotella intermedia 17. This is a clinical strain isolated from the periodontal pocket and is one of the most studied of the Prevotella intermedia strains. The genome has facilitated gene discovery in a number of studies [23-25]. DOE Joint Genome Institute also submitted the draft genome sequence of the type strain, Prevotella intermedia ATCC 25611, a strain isolated from empyema. We isolated the clinical strain Prevotella intermedia ZT from the infected root canal of a Chinese patient with chronic apical abscess and obtained its draft genome. To further understand the general character of the genus Prevotella we also compared this strain with other taxa of the genus Prevotella.

\section{Results and discussion}

The general feature of the Prevotella intermedia ZT genome 89 contigs of Prevotella intermedia ZT genome were assembled (Figure 1) while 91 singletons longer than $200 \mathrm{bp}$ remained. 2165 ORF were predicted. The genome and annotations (BioProject ID: PRJNA208776; accession: ATMK00000000; BioSample SAMN02212661) have been submitted to NCBI.

\section{Genomic comparison of virulence factors in Prevotella intermedia strains \\ General features of genome comparison}

We aligned the assembled contigs of Prevotella intermedia ZT with the published $P$. intermedia 17 genome (Accession: PRJNA163151; GenBank: CP003502.1 and CP003503.1) and Prevotella intermedia ATCC 25611 DSM 20706 (Accession: PRJNA185645; RefSeq: NZ_ JAEZ00000000.1) using MUMmer3.23. One average, $88 \%$ of the genome is matched with the weighed identity of $96.2 \%$ between any two genomes.

We clustered the genes of Prevotella intermedia ZT, 17 and ATCC 25611 and analyzed the COG classification. The numbers of core genes of the analyzed Prevotella intermedia strains are 1602, 1629 and 1602 (some clusters contains more than one genes from a genome) respectively, which is about $72 \%$ of all the genes encoded by each strain (Figure 2).

\section{Genes involved in adaptation and pathogenicity}

The pathogenicity of anaerobic oral microbes usually initiates with the attachment and invasion of the host tissue. Pathogen will then break down certain cells or proteins of the host and later interfere with the immune system, stimulating inflammatory reaction [26]. Most of the genes involved in pathogenicity are shared by the three strains, but there are some strain-specific genes that contribute to adaption to niche and pathogenicity.

\section{Adhesion and attachment}

Adhesion to the surface of the tooth or mucosa is the first step and an essential requirement for pathogen survival and pathogenicity in oral cavity or empyema. The structure of cell envelope is the foundation of adhesion 


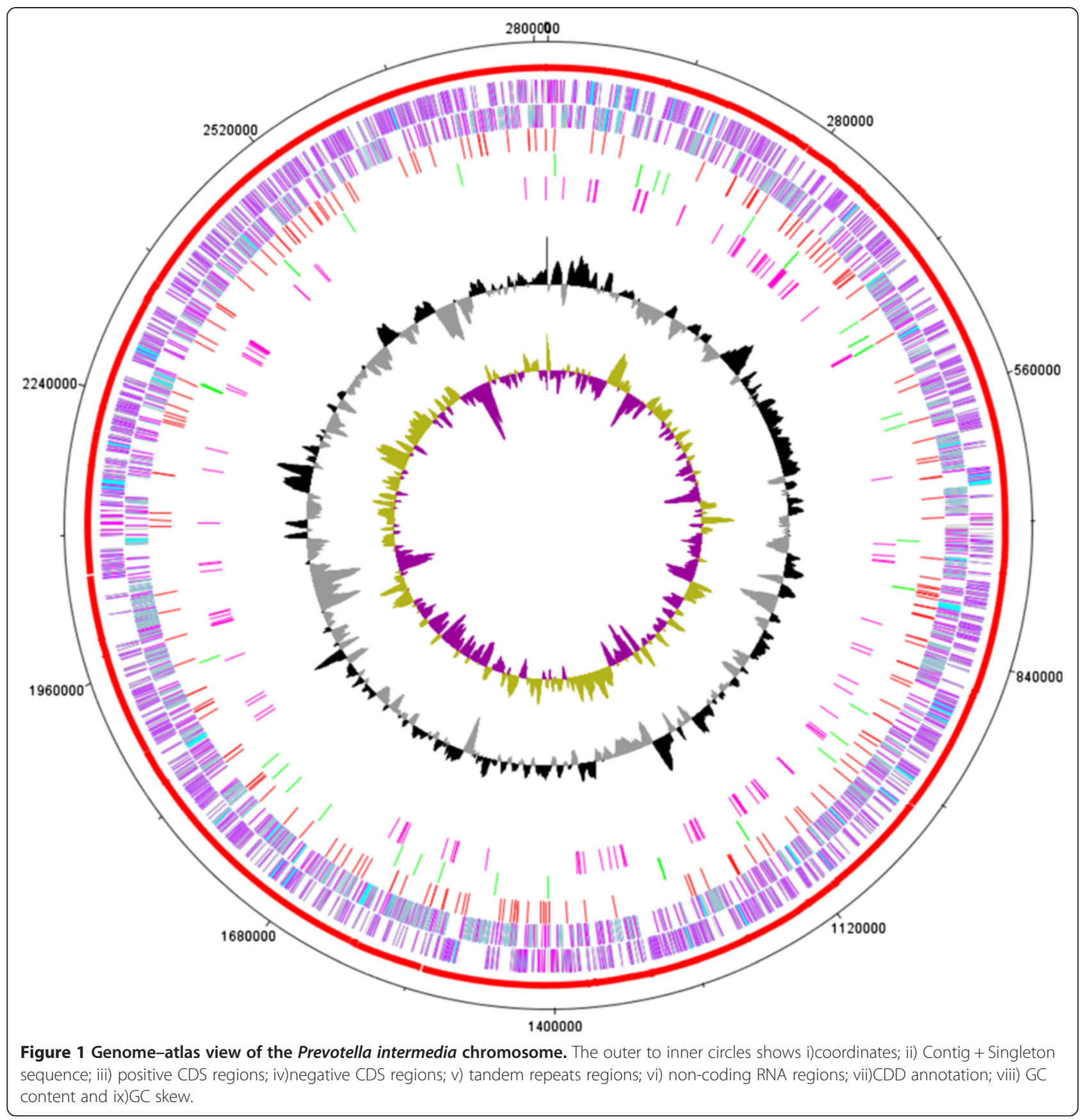

to host tissue and surrounding microbes. $85 \%$ of the homologous gene clusters predicted to be involved in cell envelope construction are conserved among the three strains while there are strain specific genes: notably, Prevotella intermedia 17 has six strain-specific glycosyltransferase clustered together.

Besides that, some gene was reported to specifically contribute to adhesion. AdpB, an ompA family gene, has been reported to be related to adhesion to host tissue. $\mathrm{AdpB}$, a $29 \mathrm{kDa}$ protein, is the first broad-spectrum ECM (extracellular matrix)-binding protein localized on the cell surface that was identified and characterized in Prevotella intermedia 17 [24]. It shared by all the strains 6 orthologous clusters of adhesin are found in Prevotella intermedia strains but only one of them is shared by all the strains. Notably, Prevotella intermedia ZT has a tandem of 4 cleaved adhesin domain protein and 3 of them are strain-specific (Table 1).

\section{Proteolysis and Bacteriocin}

Protease or peptidase is one of the major virulence factors of Prevotella intermedia. Besides its role in 

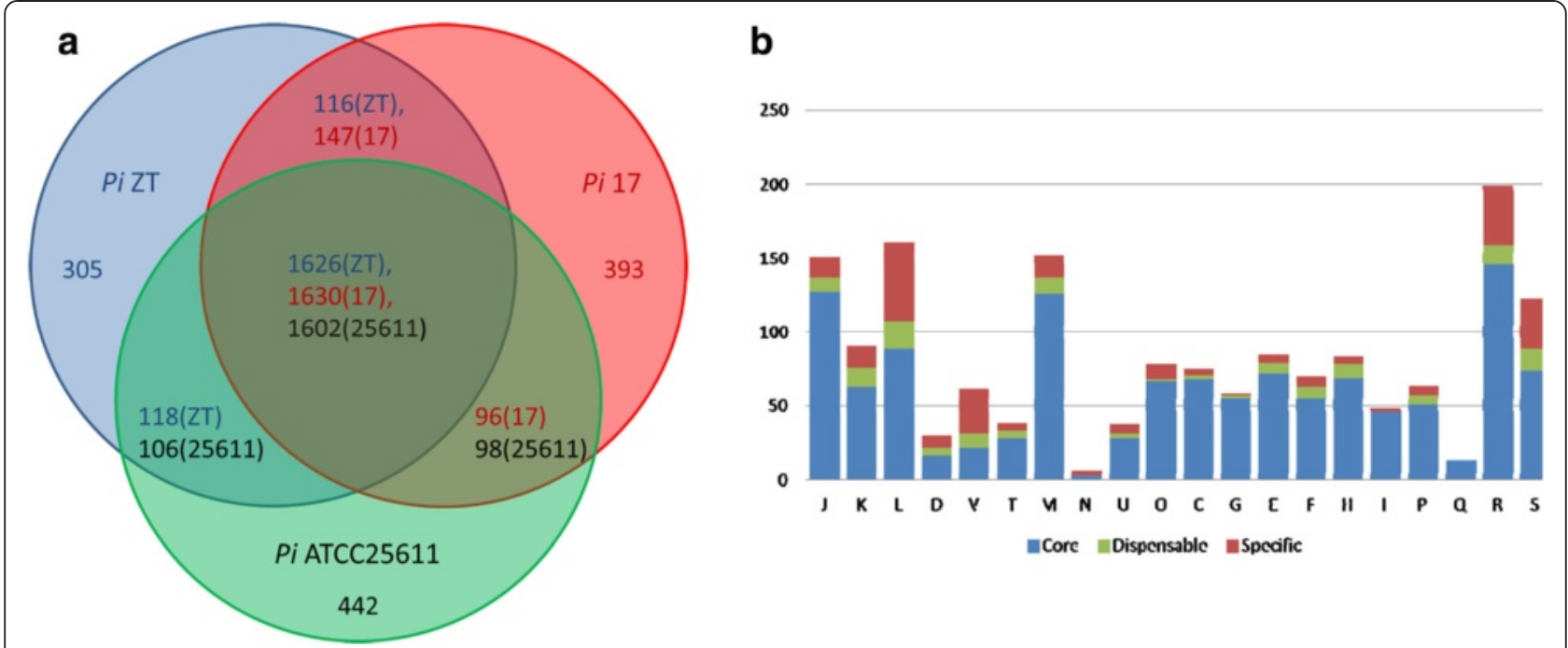

Figure 2 Comparison of Prevotella intermedia ZT, 17 and ATCC 25611. a, The Vann's diagram of gene content difference of the three strains. b. the COG classification of the homologous gene clusters of Prevotella intermedia ZT, 17 and ATCC 25611. Core: Shared by all the three genomes; dispensable: Shared by part of the genomes; specific: strain specific. Abbreviation, function description and is shown as follows : D, Cell division and chromosome partitioning; M, Cell envelope biogenesis, outer membrane; N, Cell motility and secretion; V, Defense mechanisms; U, Intracellular trafficking and secretion; O, Posttranslational modification, protein turnover, chaperones; T, Signal transduction mechanisms; L, DNA replication, recombination, and repair; K, Transcription; J, Translation, ribosomal structure and biogenesis; E, Amino acid transport and metabolism; G, Carbohydrate transport and metabolism; H, Coenzyme metabolism; C, Energy production and conversion; P, Inorganic ion transport and metabolism; I, Lipid metabolism; F, Nucleotide transport and metabolism; Q, Secondary metabolites biosynthesis, transport, and catabolism; S, Function unknown; $\mathrm{R}$, General function prediction only.

degrading the host tissue, proteolysis is also an important part of the signaling pathway involved in various pathologies including inflammatory diseases [27,28]. Previous studies have shown that Prevotella intermedia degrades proteins such as immunoglobulin and LPS- binding protein (LBP) to impede the defense of the host [29-31].

A large proportion of the proteases locate in cytoplasm and participate in the basic metabolism such as amino acid metabolism and posttranslational modification

Table 1 The existence of adpB in the Prevotella intermedia strains and specific genes of cell envelope synthesis and adhesion

\begin{tabular}{|c|c|c|c|}
\hline Pi ZT & Pi 17 & Pi ATCC 25611 & Annotation \\
\hline 127001 & 387132175 & 2515940236 & $\begin{array}{l}\text { OmpA/adpB/Outer membrane protein and related } \\
\text { peptidoglycan-associated (lipo)proteins }\end{array}$ \\
\hline 115010 & - & - & Glycosyl transferase \\
\hline 105038 & - & - & Energy transducer TonB \\
\hline 115011 & - & - & Nucleotide sugar dehydrogenase \\
\hline 107096 & - & - & Peptidase S41 \\
\hline- & 387133452 & - & Glycosyltransferase family protein \\
\hline- & 387133451 & - & Glycosyltransferase, group 2 family protein \\
\hline- & 387133459 & - & Glycosyltransferase, group 1 family protein \\
\hline- & 387133461 & - & Sugar transferase \\
\hline- & 387133454 & - & Glycosyltransferase, group 1 family protein \\
\hline 118017 & 387131650 & 2515939909 & Cleaved adhesin domain. \\
\hline 115038 & - & 2515938020 & Cleaved adhesin domain./CARDB. \\
\hline- & 387133158 & 2515938585 & Cleaved adhesin domain./CARDB. \\
\hline 104072 & - & - & Cleaved adhesin domain protein \\
\hline 104074 & - & - & Cleaved adhesin domain protein \\
\hline 104075 & - & - & Cleaved adhesin domain protein \\
\hline
\end{tabular}

The genes in the same raw belong to one homologous cluster with the threshold of e value $10^{-10}$, coverage $80 \%$ and identity $60 \%$. 
(Figure 3). Interestingly, Among the 24 strain specific proteases, 6 of them are bacteriocin or bacteriocin/lantibiotic exporters. The bacteriocins and lantibiotics also show great strain-specificity: except the two homologous cluster of lantibiotic ABC transporter and one cluster of bacteriocin, the rest of them are all strain specific. Bacteriocin and lantibiotic inhibit the grows of similar or closely bacterial strains. This indicate that the competition with the surrounding strains in the same niche, instead of breaking down proteins in the host tissues as we previously expected, may drive the evolution of Prevotella intermedia (Table 2).

\section{Iron uptake}

Lysis of erythrocytes and degradation of hemoglobin are the major iron source of Prevotella intermedia [32]. This process begins with the accumulation of erythrocytes. According to a previous study, fimbriae of Prevotella intermedia induce hemagglutination [33], and the gene encoding hemagglutinin has been isolated [34,35]. Hemagglutinin, or mannosyl-glycoprotein endobeta- $\mathrm{N}$-acetylglucosaminidase, induces the aggregation of erythrocyte

Prevotella intermedia also has hemolytic activity [34,36-39]. When Prevotella intermedia is incubated in medium with rabit/sheep/humam red blood cells, betahemolytic zones are formed. The hemolytic activity reaches its maximum in weak acid condition [36]. The gene encoding hemolysin, the protein responsible for hemolytic activity, has been isolated and characterized [38]. Hemolysin gene is also identified in all Prevotella intermedia strains.
Its homologous genes with the same/similar function are found in a wide range of Prevotella taxa

As to degredation of hemoglobin, Prevotella intermedia 17 encodes a cysteine protease named Interpain A (InpA), which breaks down oxyhaemoglobin by oxidating it to aquomethaemoglobin (in which the heam iron is oxidated to the $\mathrm{Fe}(\mathrm{III})$ state and has $\mathrm{H}_{2} \mathrm{O}$ as sixth co-ordinate ligand) at pH6.0. Aquemethaemoglobin is further completely broken down and hame is released to generate black-colored Fe(III) protoporphyrin IX pigment $[40,41]$. Interpain A also disturbs immune reaction of the host by degrading complement factor C3 [42]. The counterpart gene is identified in other Prevotella intermedia strains and its homologous proteins are found in Prevotella nigrescens and Prevotella pallens.

Prevotella intermedia strains share 2 copies of heminbinding protein genes and a hemin receptor gene. Although PSORTb [43] fails to give any explicit prediction of the location of the two hemin-binding protein, their homologous genes in other Prevotella spp. are annotated to locate on the membrane. Hemin-binding protein identified in oral pathogen Porphyromonas gingivalis [44,45] and Treponema denticola [46] is a membrane protein that facilitates the uptake of hemin and the deletion of it will retard the growth of the bacteria in the iron-restricted media.

The whole pathway is intact in all the three strains. Although some genes with on the pathway are annotated to be hypothetic protein, they have more than $95 \%$ whole length identity with the genes annotated to be in the iron uptake pathway (Table 3).

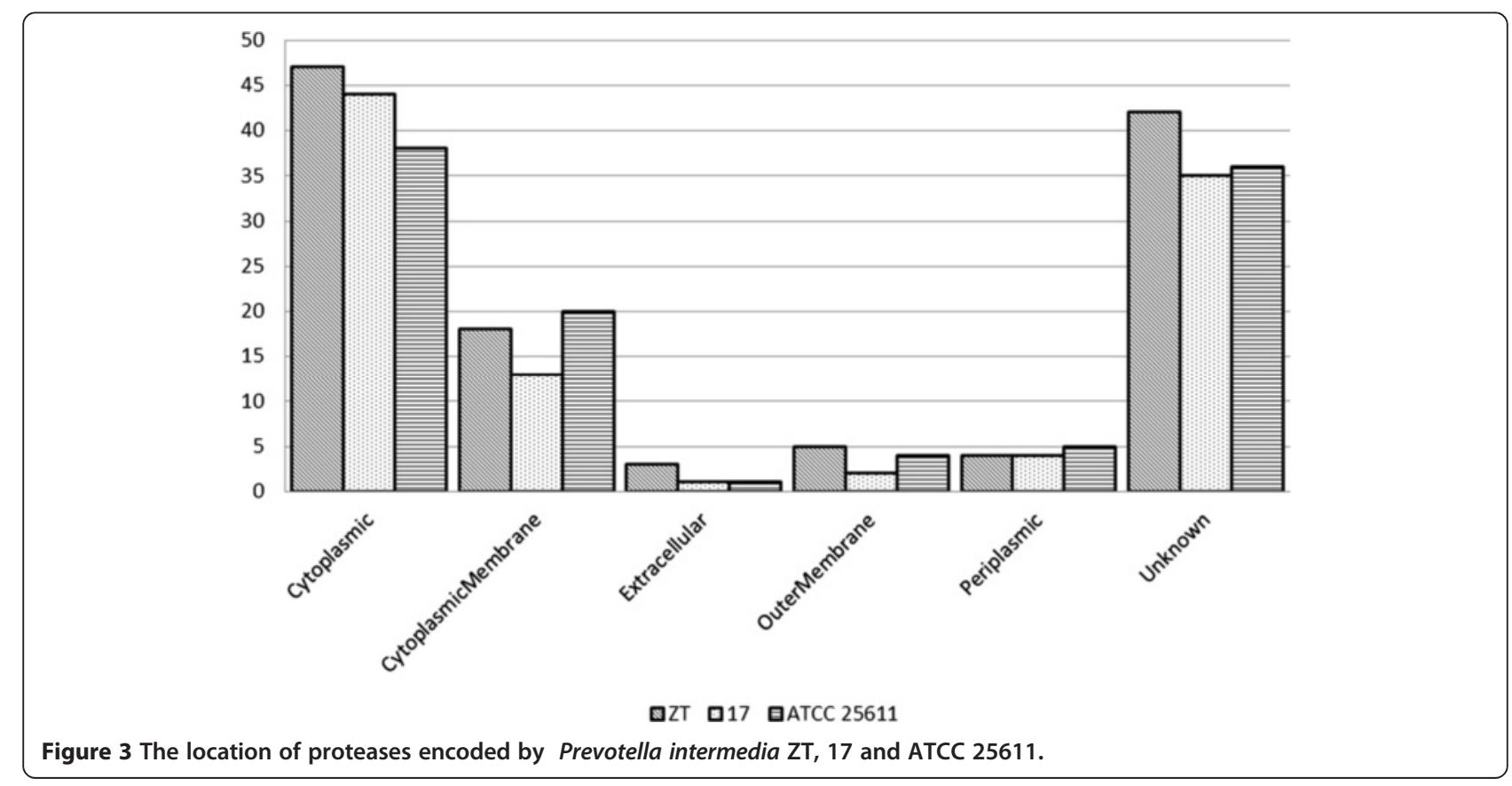


Table 2 Comparison of proteases and genes related with bacteriocin in the Prevotella intermedia strains

a. The specific proteases of the Prevotella intermedia strains. Note the genes involved in bacteriocin

\begin{tabular}{|c|c|c|c|}
\hline$\overline{P i Z T}$ & Pi 17 & Pi ATCC 25611 & Annotation \\
\hline 107096 & - & - & Peptidase S41 \\
\hline 108006 & - & - & CAAX protease \\
\hline 108029 & - & - & Hypothetical protein \\
\hline 114036 & - & - & Peptidase M23 \\
\hline 118026 & - & - & DJ-1/Pfpl family protein \\
\hline 126005 & - & - & ATP-dependent serine protease \\
\hline 134012 & - & - & Hypothetical protein \\
\hline 134014 & - & - & Hypothetical protein \\
\hline 139007 & - & - & Peptidase \\
\hline 142001 & - & - & Bacteriocin \\
\hline 142003 & - & - & Peptidase inhibitor 19 domain protein \\
\hline 142004 & - & - & Bacteriocin \\
\hline- & 387131655 & - & Putative bacteriocin \\
\hline- & 387131961 & - & Putative bacteriocin \\
\hline- & 387132117 & - & Putative caudovirus prohead protease \\
\hline- & 387132819 & - & Putative caudovirus prohead protease \\
\hline- & 387133830 & - & Hypothetical protein PIN17_A1967 \\
\hline- & 387132710 & - & Peptidase, M23 family \\
\hline- & - & 2515938070 & $\begin{array}{l}\text { ABC-type bacteriocin/lantibiotic exporters, contain an } \mathrm{N} \text {-terminal } \\
\text { double-glycine peptidase domain }\end{array}$ \\
\hline- & - & 2515938139 & Hypothetical protein \\
\hline- & - & 2515938401 & Hypothetical protein \\
\hline- & - & 2515939618 & Membrane-bound metallopeptidase \\
\hline- & - & 2515939771 & $\begin{array}{l}\text { ABC-type bacteriocin/lantibiotic exporters, contain an N-terminal } \\
\text { double-glycine peptidase domain }\end{array}$ \\
\hline- & - & 2515940199 & $\begin{array}{l}\text { Trypsin-like serine proteases, typically periplasmic, contain } \\
\text { C-terminal PDZ domain }\end{array}$ \\
\hline \multicolumn{4}{|c|}{ b. The genes involved in bacteriocin in the Prevotella intermedia strains } \\
\hline 103143 & 387132014 & 2515939098 & Lantibiotic ABC transporter \\
\hline $120031 ; 140001 ; 141001$ & - & $2515939774 ; 2515938065$ & $\begin{array}{l}\text { ABC-type bacteriocin/lantibiotic exporters, contain an N-terminal } \\
\text { double-glycine peptidase domain }\end{array}$ \\
\hline- & - & 2515938070 & $\begin{array}{l}\text { ABC-type bacteriocin/lantibiotic exporters, contain an N-terminal } \\
\text { double-glycine peptidase domain }\end{array}$ \\
\hline- & - & 2515939771 & $\begin{array}{l}\text { ABC-type bacteriocin/lantibiotic exporters, contain an N-terminal } \\
\text { double-glycine peptidase domain }\end{array}$ \\
\hline 120005 & $387131983 ; 387131657$ & 2515939795; 2515939797 & Bacteriocin/Subtilisin-like serine proteases \\
\hline- & 387131961 & - & Putative bacteriocin \\
\hline- & 387131655 & - & Putative bacteriocin \\
\hline 142001 & - & - & Bacteriocin \\
\hline 142002 & - & - & Bacteriocin \\
\hline 142004 & - & - & Bacteriocin \\
\hline
\end{tabular}

\section{Drug resistance}

Prevotella intermedia strains are predicted to encode different copies of beta-lactamases and multidrug/efflux transporters, which provide resistance to antibiotics. Genes of beta-lactamases and multidrug/efflux transporters are shared by almost all the three strains although in two of the homologous clusters, some of the genes are not annotated to be beta-lactamases despite of the more than $95 \%$ whole length identity with betalactamase gene (Table 4).

\section{Other feature related to virulence}

Dipeptidyl peptidase IV(DPP4) are also found in the all the three Prevotella intermedia strains. DPP4 is a 
Table 3 The iron uptake pathway of Prevotella strains

\begin{tabular}{llll}
\hline Pi ZT & Pi 17 & Pi ATCC 25611 & Annotation \\
\hline 103136 & 387131911 & 2515939091 & Hemagglutinin/mannosyl-glycoprotein endo-beta-N-acetylglucosaminidase/Muramidase (flagellum-specific) \\
\hline 103039 & 387131802 & 2515938961 & Hemolysins/membrane protein, PF01595 family/Hemolysins and related proteins containing CBS domains \\
\hline 111047 & $387133496^{*}$ & $2515938788^{*}$ & hemolysin/gliding motility-associated protein GldE \\
\hline 103117 & 387131889 & $2515939069^{*}$ & Hemolysin secretion protein D/HlyD family secretion protein/Multidrug resistance efflux pump \\
\hline 109025 & $387132323^{*}$ & 2515939567 & Hemolysin/acetyltransferase (GNAT) domain protein/Putative hemolysin \\
\hline 118018 & $387131649^{*}$ & $2515939908^{*}$ & Interpain A/Peptidase C10 family. \\
\hline 107033 & $387132883^{*}$ & $2515939291^{*}$ & Hemin-binding protein/hypothetical protein/Uncharacterized secreted protein \\
\hline 105015 & $387133319^{*}$ & $2515939710^{*}$ & Hemin-binding protein/hypothetical protein/hypothetical protein \\
\hline 105006 & $387133309^{*}$ & $2515938428^{*}$ & Hemin receptor/hypothetical protein/hypothetical protein \\
\hline 111047 & $387133496^{*}$ & $2515938788^{*}$ & Hemolysin/gliding motility-associated protein GldE \\
\hline 103136 & 387131911 & 2515939091 & Hemagglutinin/mannosyl-glycoprotein endo-beta-N-acetylglucosaminidase/Muramidase (flagellum-specific) \\
\hline 103039 & $387131802^{*}$ & 2515938961 & Hemolysins/membrane protein, PF01595 family/Hemolysins and related proteins containing CBS domains \\
\hline 111047 & $387133496^{*}$ & $2515938788^{*}$ & Hemolysin/gliding motility-associated protein GldE \\
\hline The genes with annotation other than hemolysin and hemin-binding are marked with *.
\end{tabular}

protease located on the surfaces of different cells across species including the genus Prevotella and also encoded by Prevotella intermedia 17 [47] and other 2 strains. Having a wide range of substrates [48], DPP4 is related to various physiological process including immune regulation, signal transduction and carcinogenesis [49-52]. A previous study showed that DPP4 from Prevotella gingivalis in gingival crevicular is associated with its pathogenicity and that Prevotella spp also has strong DPP activity [53]. This evidence points to an important role for DPP4 in pathogenicity.

Hypothetical virulence factor BrkB (103055), a 448amino acid protein, was also found shared by the three strains. Virulence factor BrkB was first identified in Bordetella pertussis and was found to be responsible for serum resistance [54]. It is also shared by the three strains.

Virulence-associated protein $\mathrm{E}$ and other toxin proteins were identified in the three strains. The strains both share several copies of homologous genes and each possesses strain-specific genes (Table 5).

\section{Recombination in Prevotella intermedia}

There are more about 130 genes predicted to have functions of replication, recombination and repair in each Prevotella intermedia genome. Recombinase, integrase and type I restriction enzymes and clusters of conjugal transfer protein genes are found in the genomes (data not shown). We also found cluster of strain-specific genes (Additional file 1) in all three strains, which are possibly the relic of previous transpositions.

Some of these clustered strain-specific genes are potentially related with adaptation and pathogenicity: Prevotella intermedia ZT has a cluster containing 1,3-betaglucan synthase regulator genes; Prevotella intermedia 17 has a cluster of $\mathrm{ABC}$ transporter genes and a cluster of glycosyltransferase genes; Prevotella intermedia ATCC 25611 has a cluster containing glycosyltransferases and $\mathrm{Na}+$-driven multidrug efflux pump and a cluster containing BC-type bacteriocin.

\section{Genomic comparison of gene content within the genus Prevotella}

In addition to the intro-species genome alignment between Prevotella intermedia ZT and Prevotella intermedia 17, we aligned the Prevotella intermedia ZT genome against the genomes of Prevotella dantalis DSM 3688 (NCBI ID: 184818), Prevotella melaninogenica ATCC 25845 (NCBI ID: 51377), Prevotella denticola F0289 (NCBI ID: 65091), Prevotella ruminicola 23 (NCBI ID: 47507) (Additional file 2). The alignments show that the genome sequences are barely matched between two different species. The matched region count only $\sim 3 \%$ of the whole genome in the nucleotide sequence. However a $\sim 49 \mathrm{~Kb}$ region that is conserved in terms of gene content and partly DNA sequence is shared by all the considered genomes (Table 6). This region located around from $56 \mathrm{~Kb}$ to $95 \mathrm{~Kb}$ of contig00007 in the draft Prevotella intermedia ZT genome sequence. It encodes DNAdirected RNA polymer, $30 \mathrm{~S}$ and $50 \mathrm{~S}$ ribosomal proteins and translation elongation factor and five tRNA. And the corresponding regions in other genomes have similar gene contents and arrangement.

\section{COG analysis}

In addition to the Cluster of Orthologous Group (COG) [55] distribution of Prevotella intermedia ZT, the Pangenome of Prevotella intermedia ZT, Prevotella intermedia 17 (NCBI ID: 163151), Prevotella dantalis DSM 3688 (NCBI ID: 184818), Prevotella melaninogenica ATCC 25845 (NCBI ID: 51377), Prevotella denticola F0289 (NCBI 
Table 4 The genes involved in drug resistance

\begin{tabular}{|c|c|c|c|}
\hline Pi ZT & Pi 17 & Pi ATCC 25611 & Annotation \\
\hline 102115 & $387133861^{*}$ & $2515938326^{*}$ & Beta-lactamase/BT1 family protein \\
\hline 103090 & 387131861 & 2515939024 & $\begin{array}{l}\text { Metallo-beta-lactamase/metallo-beta-lactamase domain-containing } \\
\text { protein/Zn-dependent hydrolases, including glyoxylases }\end{array}$ \\
\hline 123014 & 387131920 & 2515940058 & Beta-lactamase \\
\hline 107038 & 387132889 & 2515939286 & Metallo-beta-lactamase \\
\hline 113052 & 387133548 & 2515938870 & Metal-dependent hydrolases of the beta-lactamase superfamily III \\
\hline 107038 & 387132889 & 2515939286 & Beta-lactamase class $C$ and other penicillin binding proteins \\
\hline 105055 & 387133360 & 2515940206 & Predicted Zn-dependent hydrolases of the beta-lactamase fold \\
\hline 103109 & 387131881 & 2515939060 & $\begin{array}{l}\text { Lipoate-protein ligase/Metal-dependent hydrolases of the } \\
\text { beta-lactamase superfamily I }\end{array}$ \\
\hline $139005^{*}$ & 387132774 & $2515939613^{*}$ & $\begin{array}{l}\text { Flavoproteins/metallo-beta-lactamase domain-containing protein/ } \\
\text { Uncharacterized flavoproteins }\end{array}$ \\
\hline 146004 & 387132848 & - & Beta-lactamase family protein \\
\hline- & - & 2515938733 & Peptidase M23 \\
\hline- & - & 2515938733 & Predicted hydrolase (metallo-beta-lactamase superfamily) \\
\hline 101036 & 387132632 & 2515939689 & ABC-type multidrug transport system, ATPase and permease components \\
\hline 101077 & 387132597 & 2515939351 & ABC-type multidrug transport system, ATPase component \\
\hline 101098 & 387132587 & 2515939361 & Predicted integral membrane protein \\
\hline $102063^{*}$ & 387133790 & $2515938274^{*}$ & Cell division protein FtsX/efflux ABC transporter permease/Cell division protein \\
\hline 102069 & 387133795 & 2515938277 & RND family efflux transporter, MFP subunit \\
\hline 102070 & 387133796 & 2515938278 & $\begin{array}{l}\text { Multidrug transporter AcrB/RND transporter, HAE1 family/The } \\
\text { (Largely Gram-negative Bacterial) Hydrophobe/Amphiphile Efflux-1 (HAE1) Family }\end{array}$ \\
\hline 102071 & 387133797 & 2515938279 & Efflux transporter, outer membrane factor (OMF) lipoprotein, NodT family \\
\hline 103067 & 387131831 & 2515938998 & ABC-type multidrug transport system, ATPase component \\
\hline 103115 & 387131887 & 2515939067 & ABC-type multidrug transport system, permease component \\
\hline 103116 & 387131888 & 2515939068 & ABC-type multidrug transport system, permease component \\
\hline $103117^{*}$ & 387131889 & 2515939069 & Hemolysin secretion protein D/Multidrug resistance efflux pump \\
\hline 104098 & 387133178 & 2515938563 & Arabinose efflux permease \\
\hline $104115^{*}$ & 387133194 & 2515938543 & GntR family transcriptional regulator/putative efflux protein, MATE family \\
\hline 106066 & 387132977 & 2515939203 & ABC-type multidrug transport system, permease component \\
\hline 106067 & 387132976 & 2515939204 & ABC-type multidrug transport system, permease component \\
\hline 106068 & 387132975 & 2515939205 & ABC-type multidrug transport system, ATPase component \\
\hline 106069 & 387132974 & 2515939206 & Multidrug resistance efflux pump \\
\hline 109065 & 387132263 & 2515940128 & ABC-type multidrug transport system, ATPase and permease components \\
\hline 110015 & 387133713 & 2515938194 & Putative efflux protein, MATE family \\
\hline 127020 & 387132192 & 2515938413 & Putative efflux protein, MATE family \\
\hline 131014 & 387133631 & 2515938091 & Putative efflux protein, MATE family \\
\hline 135009 & 387133260 & 2515938480 & Putative efflux protein, MATE family \\
\hline 148002 & $387132059 ; 387133141$ & $\begin{array}{l}\text { 2515939982; } 2515939845 ; \\
2515939937\end{array}$ & ABC-type multidrug transport system, ATPase and permease components \\
\hline $103118^{*}$ & 387131890 & $2515939070^{*}$ & Alkaline protease/outer membrane efflux protein/Outer membrane protein \\
\hline $119009^{*}$ & 387132415 & 2515940112 & $\begin{array}{l}\text { Membrane protein/efflux ABC transporter permease/ABC-type } \\
\text { transport system, involved in lipoprotein release, permease component }\end{array}$ \\
\hline 101088 & 387133824 & - & Multidrug DMT transporter permease \\
\hline 118030 & 387132859 & - & MATE efflux family protein \\
\hline- & $387133140 ; 387132058$ & $\begin{array}{l}2515939938 ; 2515939981 ; \\
2515939846\end{array}$ & ABC-type multidrug transport system, ATPase and permease components \\
\hline- & 387132973 & 2515939207 & Outer membrane efflux protein/Outer membrane protein \\
\hline
\end{tabular}


Table 4 The genes involved in drug resistance (Continued)

\begin{tabular}{|c|c|c|c|}
\hline- & 387131718 & - & Sugar efflux transporter for intercellular exchange \\
\hline- & - & 2515938842 & $\mathrm{Na}+$-driven multidrug efflux pump \\
\hline - & - & 2515939209 & ABC-type multidrug transport system, ATPase component \\
\hline
\end{tabular}

The genes with annotation other than beta-lactamase and multidrug efflux transporter marked with *.

ID: 65091), Prevotella ruminicola 23 (NCBI ID:47507) was also annotated.

Unlike the COG analysis of a single genome, COG analysis of pan-genome gives the number of gene clusters classified with certain annotation instead of the number of genes. The COG result is stratified based on the gene cluster level given by PGAP1.1. The gene cluster level is the number of the genome that distribute to a certain cluster. If a cluster has the cluster level of $n$, it has the genes from $n$ genome(s). Core clusters consist of genes from all the analyzed genomes (in our study, they have the cluster level of 6). Strain-specific clusters consist of genes from only one genome and have the cluster level of 1 . The rest are dispensable clusters. Wholecluster presents the gene function distribution of all the genes in the pan-genome, namely, the sum of core, dispensable and strain-specific clusters.

As expected, Prevotella intermedia ZT has a similar COG distribution with the whole-cluster of pan-genome (Figure 4). The function class with relatively high number of gene clusters in the pan-genome also has relatively high number of genes in Prevotella intermedia ZT genome and verse visa. As taxa of the same species, they reasonably share some common features.

The more striking feature of the analyzed taxa is the specific genes. For every COG class, the major of the clusters are strain-specific (Figure 5) which accords with the results drown from the mathematics model that the genus Prevotella has a considerable number of strainspecific genes.

The genes classified into the "intracellular trafficking, secretion and vesicular transport" is absent in the core genome but enriched in strain-specific cluster. The high species specificity of secretion function in the genus Prevotella indicates the high specificity of the interaction between Prevotella taxa and its niche.

There is no gene in "cell motility" class in the core genome either. However, considering the fact that "cell motility" has the least genes in every single genome and that KEGG pathway analysis shows that the genus Prevotella generally has no or only one gene in flagella synthesis pathway, the absence of "cell motility" gene in the core genome should be due to the low motility and probably the absence of flagella of the genus Prevotella. We assume that the genus Prevotella make use of other structures or the help of the surrounding microbe populations to attach on the surface of the oral cavity and invade into the abscess tissue.

\section{Pan-genome analysis and gene distribution mathematics model}

Pan-genome is originally used to describe the core genome of all taxa of a species typically the bacteria and archaea species [56]. Considering the similarity between different species of the genus Prevotella, the term pangenome is used to describe the essential genes of the

Table 5 Other genes involved in drug resistance

\begin{tabular}{|c|c|c|c|}
\hline Pi ZT & Pi 17 & Pi ATCC 25611 & Annotation \\
\hline 132012 & 387133613 & 2515938073 & Peptidase S9/Dipeptidyl aminopeptidases/acylaminoacyl-peptidases \\
\hline 103055 & 387131819 & $2515938987^{*}$ & Hypothetical virulence factor BrkB/ Predicted membrane protein \\
\hline $\begin{array}{l}118023 ; 149001 ; \\
116050 ; 101089\end{array}$ & $\begin{array}{l}387132842 ; 387133147 ; \\
387133822\end{array}$ & $2515938146 ; 2515939042$ & $\begin{array}{l}\text { Virulence-associated protein ENirulence-associated protein } \\
\text { E/Predicted P-loop ATPase and inactivated derivatives }\end{array}$ \\
\hline 122010 & 387132071 & 2515939824 & Toxin HipA/HipA-like C-terminal domain./HipA-like N-terminal domain \\
\hline 138006 & - & 2515940043 & Toxin Fic/Virulence protein \\
\hline 106093 & - & - & Virulence-associated protein E \\
\hline- & 387132952 & - & Virulence-associated protein E \\
\hline- & - & 2515939988 & Virulence-associated protein E. \\
\hline- & - & 2515939916 & Virulence protein \\
\hline- & 387131936 & - & Toxin-antitoxin system, toxin component, Fic domain protein \\
\hline- & - & 2515939808 & Addiction module toxin, RelE/StbE family \\
\hline- & 387131936 & - & Toxin-antitoxin system, toxin component, Fic domain protein \\
\hline
\end{tabular}

The genes with annotation other than beta-lactamase and multidrug efflux transporter marked with *. 
Table 6 The conserved region across different taxa

\begin{tabular}{lll}
\hline Strains & Contig or Chromosome & Range \\
\hline P. intermedia ZT & contigs00007 & 56Kb to 95Kb \\
\hline P. intermedia 17 & gi|387132089|ref|NC_017861.1| & 986Kb to $1024 \mathrm{~kb}$ \\
\hline P. dentalis & gi|433651058|ref|NC_019960.1| & $274 \mathrm{~kb}$ to $326 \mathrm{~kb}$ \\
\hline P. denticola & gi|327312315|reff|NC_015311.1| & $1066 \mathrm{~Kb}$ to $1110 \mathrm{~Kb}$ \\
\hline P. melaninogenica & gi|302344773|ref|NC_014370.1| & $412 b$ to $457 \mathrm{~kb}$ \\
\hline P. ruminicola & gi|294672793|ref|NC_014033.1| & 2570Kb to $2522 \mathrm{~Kb}$ \\
\hline
\end{tabular}

genus Prevotella. For the sake of data validity we use only the completely sequenced Prevotella taxa and Prevotella intermedia ZT to perform the pan-genome analysis. The completely sequenced genomes are Prevotella intermedia 17 (NCBI ID: 163151), Prevotella dantalis DSM 3688 (NCBI ID: 184818), Prevotella melaninogenica ATCC 25845 (NCBI ID: 51377), Prevotella denticola F0289 (NCBI ID: 65091), Prevotella ruminicola 23 (NCBI ID: 47507).

The genes from the six genomes were clustered with PGAP1.1 and only bidirectional best hits were further analyzed. The genus Prevotella has about 165-170 core genes, that is, the genes shared by all the analyzed taxa. This number slightly varies between different genomes because of gene duplications or homology. Each genome has strain-specific genes ranging from 1418 to 2763 (Table 7).

Venn diagram is usually used to show the distribution of conserved and strain-specific genes. However it will be too fuzzy when more than three genomes are to be considered. Therefore, we take advantage of the mathematics model provided by the PGAP to explicit the gene distribution of the genus Prevotella.

The mathematics model exhausts the pan-genome and core genome size generated from every possible combination of genomes and fit the data with a power model for pan-genome and an exponent model for coregenome:

$$
\begin{aligned}
& y=a x^{b}+c(a, \quad b, \quad c>0) \\
& z=m e^{-n x}+k(m, \quad n, \quad k>0)
\end{aligned}
$$

where $y$ denotes the number of genes in the pangenome; $z$ denotes the number of genes in the core genome; $x$ denotes the genome number and $a, b, c, m, n, k$ are the fitting parameter.

In the pan-genome model, parameter $b$ is less than 1 because the contribution of each extra single genome added to the pan-genome analysis tends decrease due to the homologous genes between the each genome. From the model we can calculate some important parameter: $y(1)$ is the average gene number of a single genome; $y(2)-y(1)$ is the average difference in gene number of the pan-genome constructed from any two genome and a single genome, that is, the average strain-specific genes in a genome when any two genomes are compared.

In the core genome model, the index of $e$ is minus because the size of the core genome decreases when more genomes are included in the pan-genome analysis. Similarly, $z(1)$ is also the average gene number of a single genome and $z(1)-z(2)$ is the average strain-specific genes in a genome when any two genomes are compared.

The mathematics model based on the six previous mentioned genomes is:

$$
\begin{aligned}
& y=1994.3 x^{0.96}+23.3 \\
& z=32442.4 \mathrm{e}^{-2.8} x+202.8
\end{aligned}
$$

In this model, $y(2)-y(1)$ is 1838.0 , which is more than half of the genes in a Prevotella genome. This mathematics model shows that on average more than half of the genes are strain-specific between any two species.

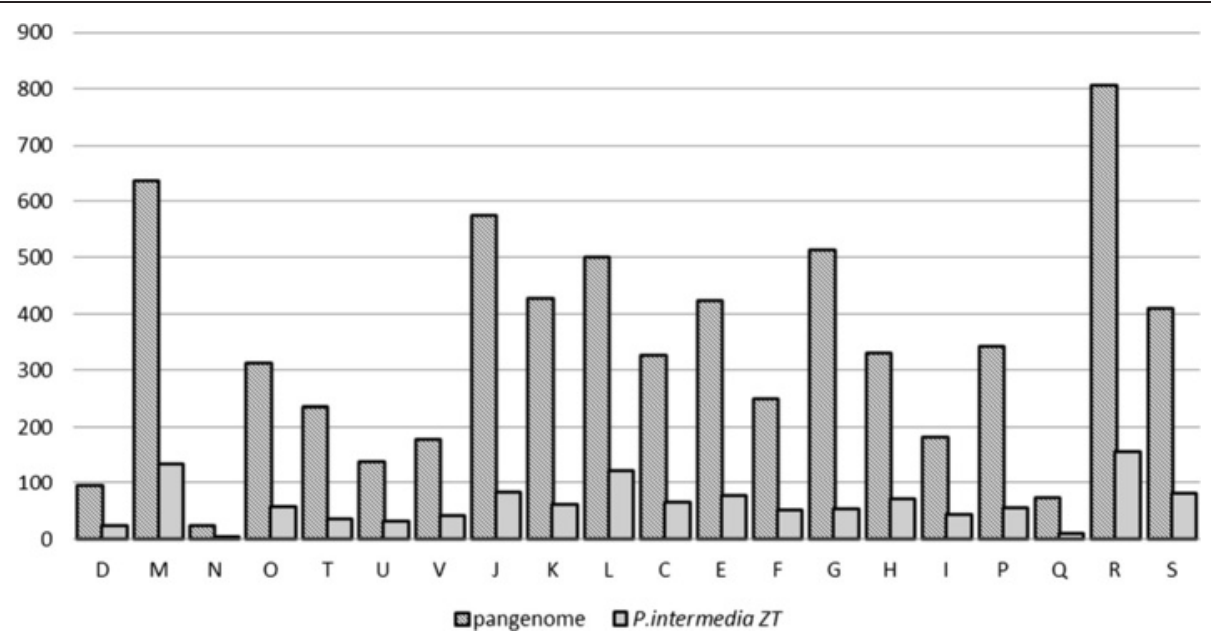

Figure 4 The COG function classification of Prevotella intermedia ZT's genes and Prevotella pan-genome gene clusters. 


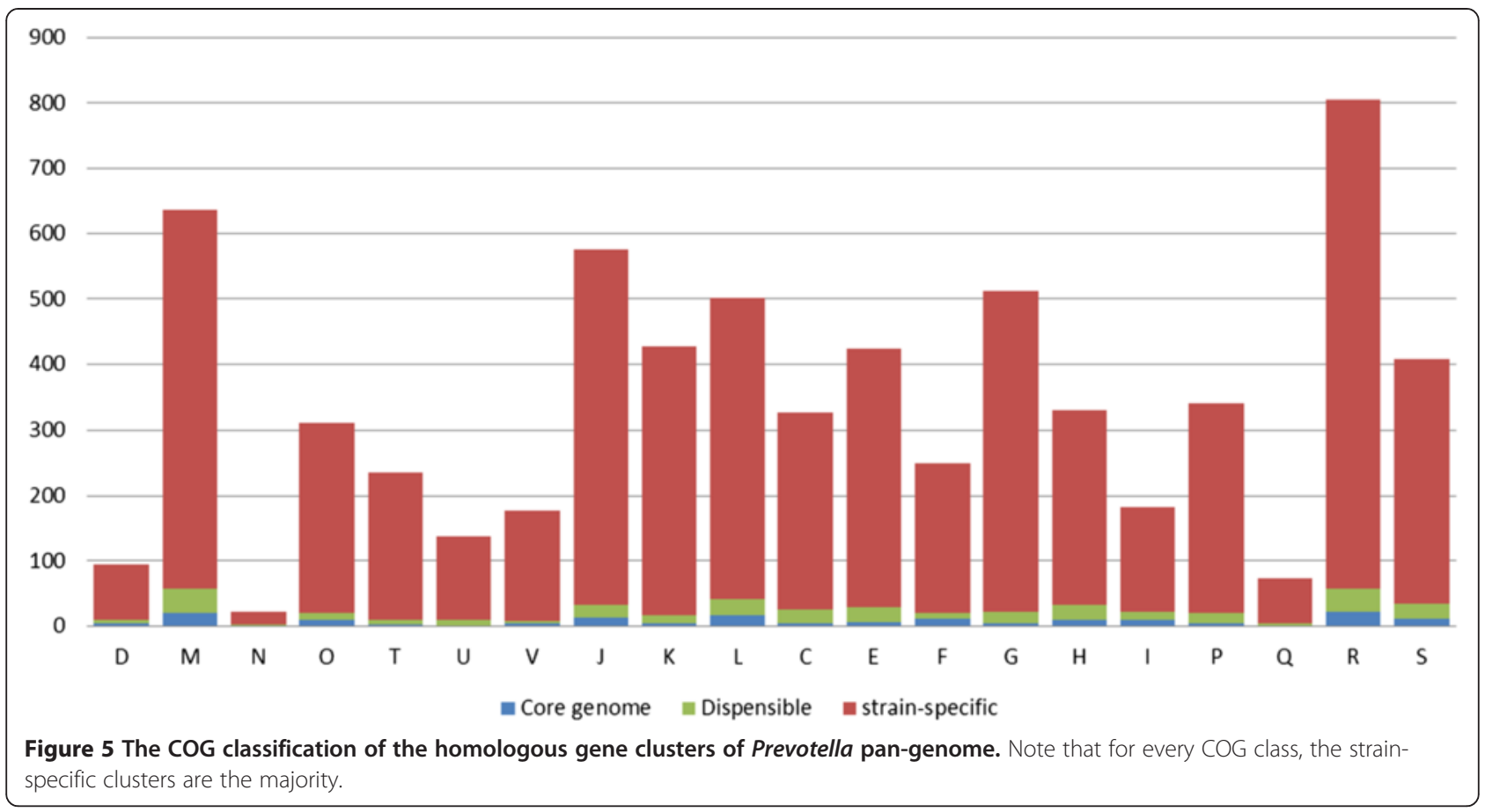

We expand the sample size and build the mathematics from 29 species of the genus Prevotella (either completely sequenced genomes or draft genomes. For each species we only chose one genome in order to prevent that the intra-species differences will not interfere the model) and draw a similar conclusion. In the new model, the average genome size is slightly larger than the previous one and the number of strain-specific genes in a single genome is notably lowered. Even so, the number of strain-specific genes between any two genomes is a considerable 1232, which is nearly half of the genes encoded by a genome.

$$
\begin{aligned}
& y=1938.9 x^{0.71}+505.9 \\
& z=3241.2 \mathrm{e}^{-0.62 \mathrm{x}}+533.5
\end{aligned}
$$

\section{Phylogenetics of the genus Prevotella}

The phylogenetic tree is a common methodology to infer the evolutionary relationship between different species. We selected among the genes that the 36 Prevotella taxa have in common (Additional file 3) and got 80 sets of homologous genes (Additional file 4). These genes are involved in carbohydrate utilization, cell component construction, genetic information process and other basic biochemistry reactions. The 80 sets of genes were used to generate a set of concatenated 34581-nt sequences to build the phylogenetic tree. Maximum likelihood and neighbor joining method were used and as expected generated almost identical results which shared the very same topology and strains of the same species were closest to each other (Figure 6).

In order to reveal the influences of niche on the evolution of core genes of the genus Prevotella, we referred to the NCBI websites and previous literature for the isolation sites of each taxon and combined the isolation sites with the phylogenetic tree. Despite the possibility that the bacteria may survive and thrive in the niches other than the isolation sites, the isolation sites at least indicate the niches which the bacteria most successfully adapt to. The majority of the analyzed taxa were isolated from oral cavity but a few taxa were isolated from

Table 7 The gene conservation distribution of each genome in the pan-genome analysis

\begin{tabular}{llllllll}
\hline Shared by taxa & $\mathbf{6}$ & $\mathbf{5}$ & $\mathbf{4}$ & $\mathbf{3}$ & $\mathbf{2}$ & $\mathbf{1}$ & Total \\
\hline Prevotella intermedia ZT & 165 & 146 & 123 & 59 & 135 & 1439 & 2067 \\
\hline Prevotella intermedia 17 & 166 & 142 & 105 & 68 & 117 & 1668 & 2266 \\
\hline Prevotella dentalis DSM 3688 & 167 & 31 & 7 & 7 & 4 & 1202 & 1418 \\
\hline Prevotella melaninogenica ATCC 25845 & 169 & 143 & 96 & 25 & 4 & 1859 & 2296 \\
\hline Prevotella denticola F0289 & 169 & 142 & 102 & 20 & 0 & 1953 & 2386 \\
\hline Prevotella ruminicola 23 & 170 & 119 & 11 & 13 & 1 & 2449 \\
\hline
\end{tabular}



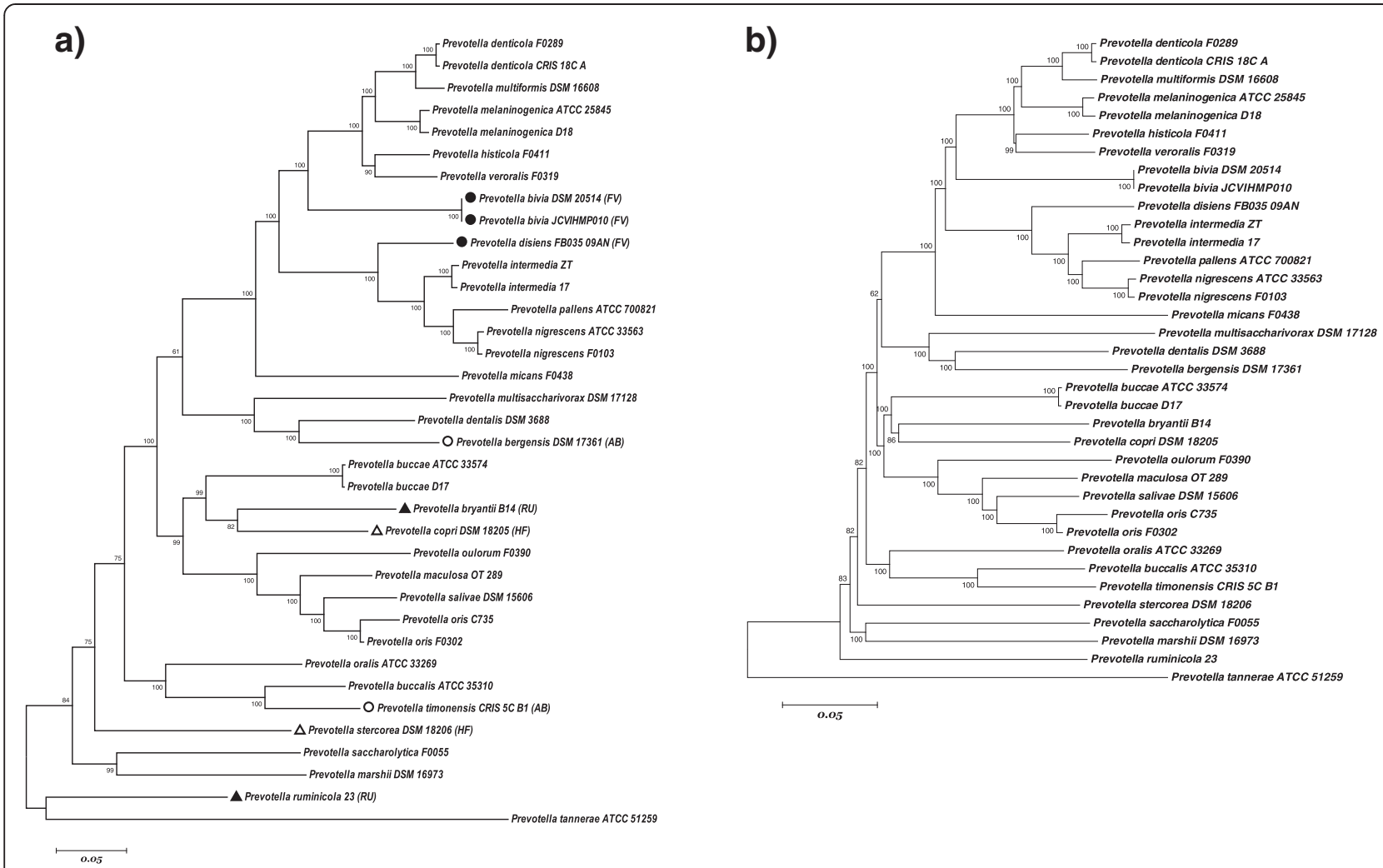

Figure 6 The phylogenetic tree of the genus Prevotella. a The phylogenetic tree build by Maximun-Likelihood Method. The isolation sites are marked on the phylogenetic tree. unmarked: oral cavity; $\triangle$ : human feces (HF); $\mathbf{\Delta}$ : rumen(RU); o: abscesses (AB); $\bullet$ : vagina (FV). b. The phylogenetic tree build by Neighbor-joining Method.

vagina (Prevotella bivia, Prevotella disiens), human feces (Prevotella stercorea, Prevotella copri), rumen and (Prevotella ruminicola, Prevotella bryantii) and abscesses (Prevotella bergensis, Prevotella timonensis). These exceptional species are scattered over different branches on the phylogenetic tree, which suggests that adapting to different niches did not play an essential role in the early evolutionary history of the genus Prevotella but accelerated the speciation in the late history.

The phylogenetic tree shows that the Prevotella intermedia has a close evolutionary relationship with Prevotella pallens, Prevotella nigrescens and Prevotella disiens. The former two species are usually isolated from oral cavity while the latter, Prevotella disiens is a pathogen that mainly exists in vagina $[57,58]$.

\section{Amino acid metabolic pathway analysis}

As a pathogen which has already adapted to protein-rich niches, Prevotella intermedia ZT lacks many biosynthesis pathways, especially those which synthesize smallmolecule nutrients such as amino acid.

The pathways of amino acid metabolism and biosynthesis are very limited. According to the KEGG pathway analysis [59], the strain Prevotella intermedia ZT does not have biosynthesis pathway for most of amino acids with only two exceptions: the synthesis of glutamine and glutamate, which plays an important role in lipopolysaccharide biosynthesis, and the biosyntheses of serine from hydroxylpyruvate. Besides that, the strain Prevotella intermedia ZT possesses some enzymes that catalyze the synthesis of amino acid from other amino acid and immediate metabolite: 1) the glycine hydroxymethyltransferase that transform glycine to serine and also the reverse reaction, 2) saccharopine dehydrogenase that degrades saccharopine into lysine, 3) L-asparaginase that transforms asparagines to aspartic acid and 4) branchedchain amino acid aminotransferase that catalyzes the reversible transformation of valine, leucine and isoleucine to their immediate metabolites 2-oxoisovalerate, 4-methyl-2-oxopentanoate and (S)-2,3-methyl-2-oxopentanoate. The previous sequenced strain Prevotella intermedia 17 shares all the amino acid metabolism pathway features with Prevotella intermedia ZT with some minor differences.

The analysis of other Prevotella taxa, that is, Prevotella dantalis DSM 3688 (NCBI ID: 184818), Prevotella melaninogenica ATCC 25845 (NCBI ID: 51377), Prevotella denticola F0289 (NCBI ID: 65091), Prevotella ruminicola 
23 (NCBI ID: 47507) shows that the genus Prevotella vary greatly in terms of amino acid metabolism. Prevotella ruminicola has the ability to generate every amino acid from other metabolites, which probably results from the carbonhydrate-rich but peptide-deficient ruminal environment. Other taxa more or less have lost the genes involved in amino acid biosynthesis and the loss of genes is most severe in Prevotella intermedia.

In terms of histidine metabolism, Prevotella intermedia has lost every gene in the histidine biosynthesis pathway but possesses a pathway to degrade histidine. Prevotella denticola and Prevotella melaninogenica have the same histidine degradation pathway as Prevotella intermedia does and they also lost most of the gene in the hisitidine biosynthesis pathway except histidinol-phosphate aminotransferase which generate L-histidinol phosphate with the translocation of an amino group from Imidazoleacetol phosphate to L-glutamate. In sharp contrast with Prevotella intermedia, Prevotella ruminicola has an intact histidine biosynthesis pathway but does not encode any enzymes to degrade hisitdine. Interestingly, Prevotella dentalis is similar with Prevotella ruminicola although it is isolated in oral cavity. It has no enzymes to metabolize histidine but a nearly full biosynthesis pathway with only two genes lost.

As to valine, leucine and isoleucine biosynthesis pathway, Prevotella intermedia, Prevotella denticola and Prevotella melaninogenica have lost all the genes except the branchedchain amino acid aminotransferase which also locates in valine, leucine and isoleucine degradation pathway. On the contrary, Prevotella ruminicola and Prevotella dentalis have a full biosynthesis pathway.

As to the synthesis of phenylalanine and tyrosine, all the analyzed taxa have some genes lost and the pathway in every genome is incomplete. The pathway can be divided into two parts: the biosynthesis of intermediate metabolite prephenate and the synthesis of phenylalanine and tyrosine from prephenate. All the taxa encode some of the enzymes involved in the biosynthesis of chorismate, the immediate metabolite that converts to prephenate reversibly. But they do not encode chorismate mutase, the enzyme that catalyzes this conversion. Both of the Prevotella intermedia strains have lost all the genes for synthesizing phenylalanine and tyrosine from prephenate while other taxa maintain two (Prevotella melaninogenica) or four genes (Prevotella dentalis, Prevotella denticola, Prevotella ruminicola).

The summary of the ability to convert other metabolite to certain amino acid is given in the Table 8. Expectedly, some conclusion about the amino acid metabolism of Prevotella intermedia drawn from the genome analysis accords with a previous bench experiment [60].

The considerable differences in amino acid biosynthesis indicate the readiness of evolution under proper

Table 8 The ability to convert other metabolites to amino acid

\begin{tabular}{|c|c|c|c|c|c|c|}
\hline Amino Acid & P. intermedia $\mathrm{ZT}$ & P. intermedia 17 & P. dentalis & P. denticola & P. melaninogenica & P.ruminicola \\
\hline Alanine & no & no & no & no & no & yes \\
\hline Arginine & no & no & yes & no & no & yes \\
\hline Asparagine & no & no & yes & yes & yes & yes \\
\hline Aspartic acid & yes & yes & no & yes & yes & yes \\
\hline Cycteine & no & no & yes & yes & yes & yes \\
\hline Glutamate & yes & yes & yes & yes & yes & yes \\
\hline Glutamine & yes & yes & yes & yes & yes & yes \\
\hline Glycine & yes & yes & yes & yes & yes & yes \\
\hline Histidine & no & no & yes & no & no & yes \\
\hline Isoleucine & no & no & yes & no & no & yes \\
\hline Leucine & no & no & yes & no & no & yes \\
\hline Lysine & yes & yes & yes & yes & yes & yes \\
\hline Methionine & no & no & yes & no & yes & yes \\
\hline Phenylalanine & no & no & yes & yes & yes & yes \\
\hline Proline & no & no & no & no & no & yes \\
\hline Serine & yes & yes & yes & yes & no & yes \\
\hline Threonine & no & no & no & no & no & yes \\
\hline Tryptophan & no & no & yes & yes & yes & yes \\
\hline Tyrosine & no & no & yes & yes & yes & yes \\
\hline Valine & no & no & yes & no & no & yes \\
\hline
\end{tabular}

The data is predicted from the KEGG pathway analysis and not yet confirmed experimentally. 
environment. The ability to synthesize amino acids, which are quite available from the host tissue, is in the process of degradation.

\section{Carbohydrate metabolic pathway and energy production} As an anaerobic microbe, the genus Prevotella only possesses a limited repertoire of enzymes for carbohydrate metabolism and energy production. The enzyme content varies from species to species. Prevotella intermedia ZT and Prevotella intermedia 17 have relatively incomplete pathways but the enzyme repertoire in Prevotella dentalis is the most reduced. Compared with the oral-isolated taxa, the rumen isolated Prevotella ruminicola has the most comprehensive repertoire of carbohydrate usage enzyme (Table 9).

Glycolysis is the first step to metabolize glucose and energy production for every species. In our study, almost all the taxa that we analyze have a functional glycolysis pathway except Prevotella dentalis, which lacks glucose-6phosphate isomerase and 6-phosphofructokinase 1. These two enzymes catalyze the second and third reaction (transform $\alpha$-D-glucose-6P to $\beta$-D-Frucose- $1,6 \mathrm{P}_{2}$ ) of the glycolysis. Prevotella dentalis also lacks glyceraldehyde 3phosphate dehydrogenase, the enzyme that catalyze the fifth reaction that transform D-glyceraldehyde 3-phosphate to 3-phospho-D-glyceroyl phosphate and several enzymes in the flanking branches of the glycolysis pathway.

In addition to glycolysis, we analyze other enzymes involved in carbohydrate metabolism. According to the KEGG pathway mapping, the genus Prevotella do not produce lactate or ethanol in cellular respiration due to the absence of the relative enzymes in the glycolysis pathway. Instead, in the pyruvate metabolism pathway, the pyruvate generated during glycolysis are the raw material for the synthesis of formate, oxaloacetate, which accords with the previous research [61]. These metabolites, especially noxious ones such as formate may contribute to the pathogenicity of the genus Prevotella.

\section{Synthesis of cell wall components}

Considering the analyzed taxa, the genus Prevotella has relatively complete, functionally conserved pathways for

Table 9 The number of genes in main pathways of carbohydrate metabolism and energy product

\begin{tabular}{llll}
\hline Taxa & $\begin{array}{l}\text { Glycolysis/ } \\
\text { Gluconeogenesis }\end{array}$ & $\begin{array}{l}\text { Starch and } \\
\text { sucrose } \\
\text { metabolism }\end{array}$ & $\begin{array}{l}\text { Pyruvate } \\
\text { metabolism }\end{array}$ \\
\hline P. intermedia ZT & 17 & 16 & 11 \\
\hline P. intermedia 17 & 17 & 16 & 12 \\
\hline$P$. dentalis & 10 & 13 & 8 \\
\hline$P$. denticola & 19 & 22 & 14 \\
\hline$P$. melaninogenica & 20 & 20 & 15 \\
\hline$P$. ruminicola & 20 & 24 & 12 \\
\hline
\end{tabular}

the synthesis of complex molecules that are used for cell material such as lipopolysaccharide and peptidoglycan.

Components such as lipopolysaccharides (LPS) [62-70] induce immunological reaction of the host. In the Prevoeolla intermedia ZT genome, we identified genes related to lipopolysaccharides synthesis. The enzymes encoded by these genes compose a nearly full pathway from primal raw material UDP-N-acetyl-D-glucosamine to $\mathrm{KDO}_{2}$-lipid IV(A) (KEGG ID: C06025) except that the 3-deoxy-Dmanno-octulosonate 8-phosphate phosphatase (KDO 8-P phosphatase) is missing. This enzyme catalyzes the transformation from 3-deoxy-D-manno-octulosonae-8P to 3deoxy-D-manno-octulosonate. Considering the economy of microbe metabolism, we conjectured that it was not likely that the whole biosynthesis of LPS should be interrupted by one single missing gene and therefore there might be other mechanism that hydrolyzed the phosphate group of 3-deoxy-D-manno-octulosonae-8P. Besides that, no enzyme on other branches of LPS synthesis has been found in this study. From the facts mentioned above, it is natural to draw the conclusion that the main component of LPS of Prevotella intermedia ZT is $\mathrm{KDO}_{2}$-lipid IV(A).

Other taxa have a similar LPS biosynthesis pathway. Generally, the Prevotella has a functional pathway to synthesize $\mathrm{KDO}_{2}$-lipid $\mathrm{IV}(\mathrm{A})$ but a few of enzymes in the branch pathway to modify the final product vary with taxa (Figure 7).

The synthesis of another important bacteria cell wall component peptidoglycan is also well equipped with a fully functional pathway (Figure 8). Compared with Prevotella intermedia 17, Prevotella intermedia ZT lacks only one gene, undecaprenyl-diphosphatase which hydrolyzes a phosphate from ditrans,octacis-undecaprenyl diphosphate. Although there are some disconnected branches in the peptidoglycan pathway, the main string of the pathway is unblocked. Besides, the blocked branches on the biosynthesis pathway indicate a corresponding biased peptidoglycan composition: The most likely major protein components of the peptidoglycan of genus Prevotella is undecaprenyl-diphospho-N-acetylmuramoyl-(N-acetylglucosamine)-L-alanyl-D-glutamyl-meso-2,6-diaminopimeloyl-D-alanyl-D-alanine (KEGG entry: C05898) and undecaprenyl-diphospho-N-acetylmuramoyl-(N-acetylglucosamine)-L-alanyl-gamma-D-glutamyl-L-lysyl-D-alanylD-alanine(KEGG entry: C05893).

\section{Conclusions}

As an important pathogen, Prevotella intermedia has long interested stomatologists and microbiologists. It is of clinical significance to study what is the potential drive of its adaptation and pathogenicity in different niches. In previous study, Prevotella intermedia 17, a clinically isolated strain from a human periodontal pocket and Prevotella intermedia ATCC 25611, the type strain isolated 


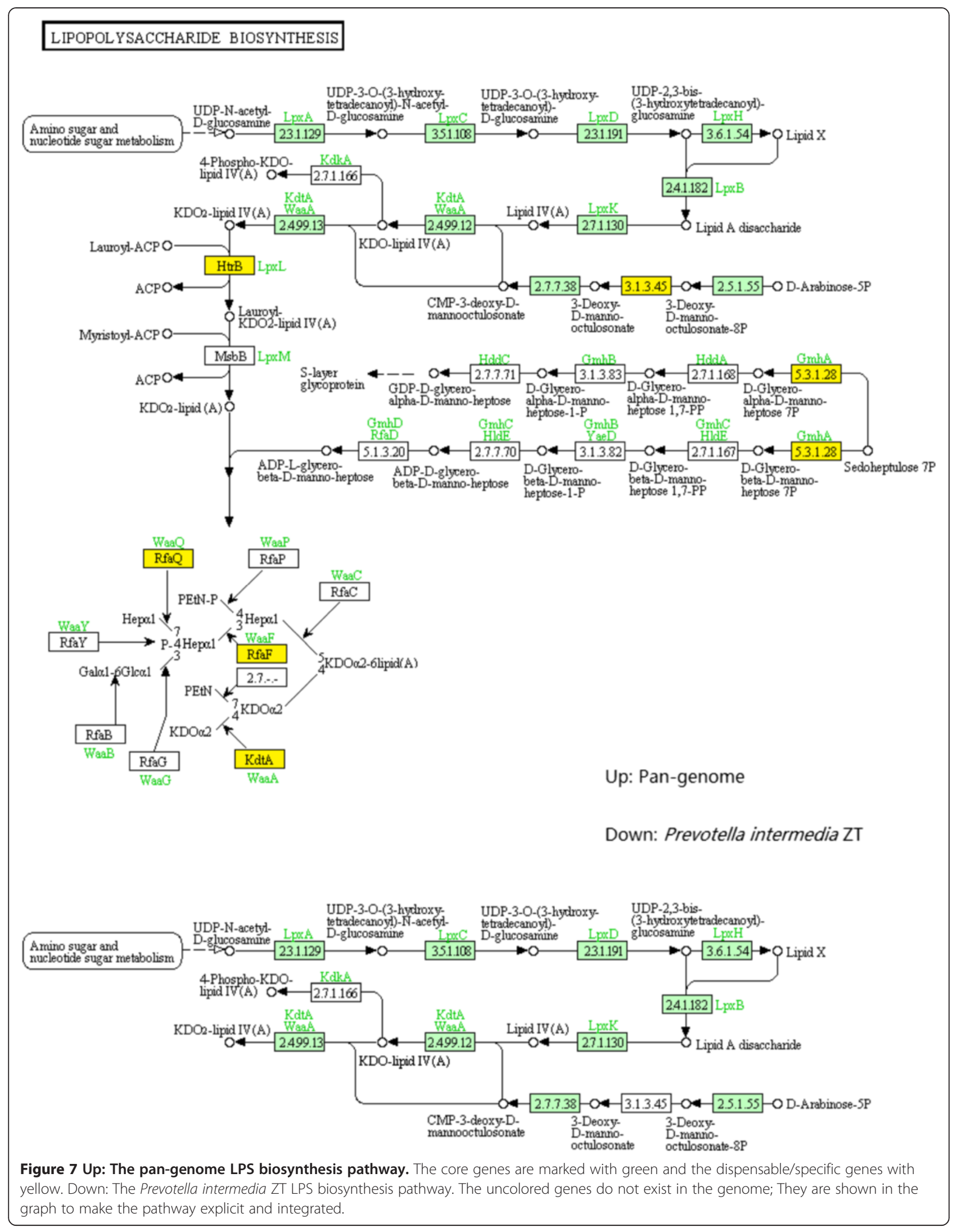



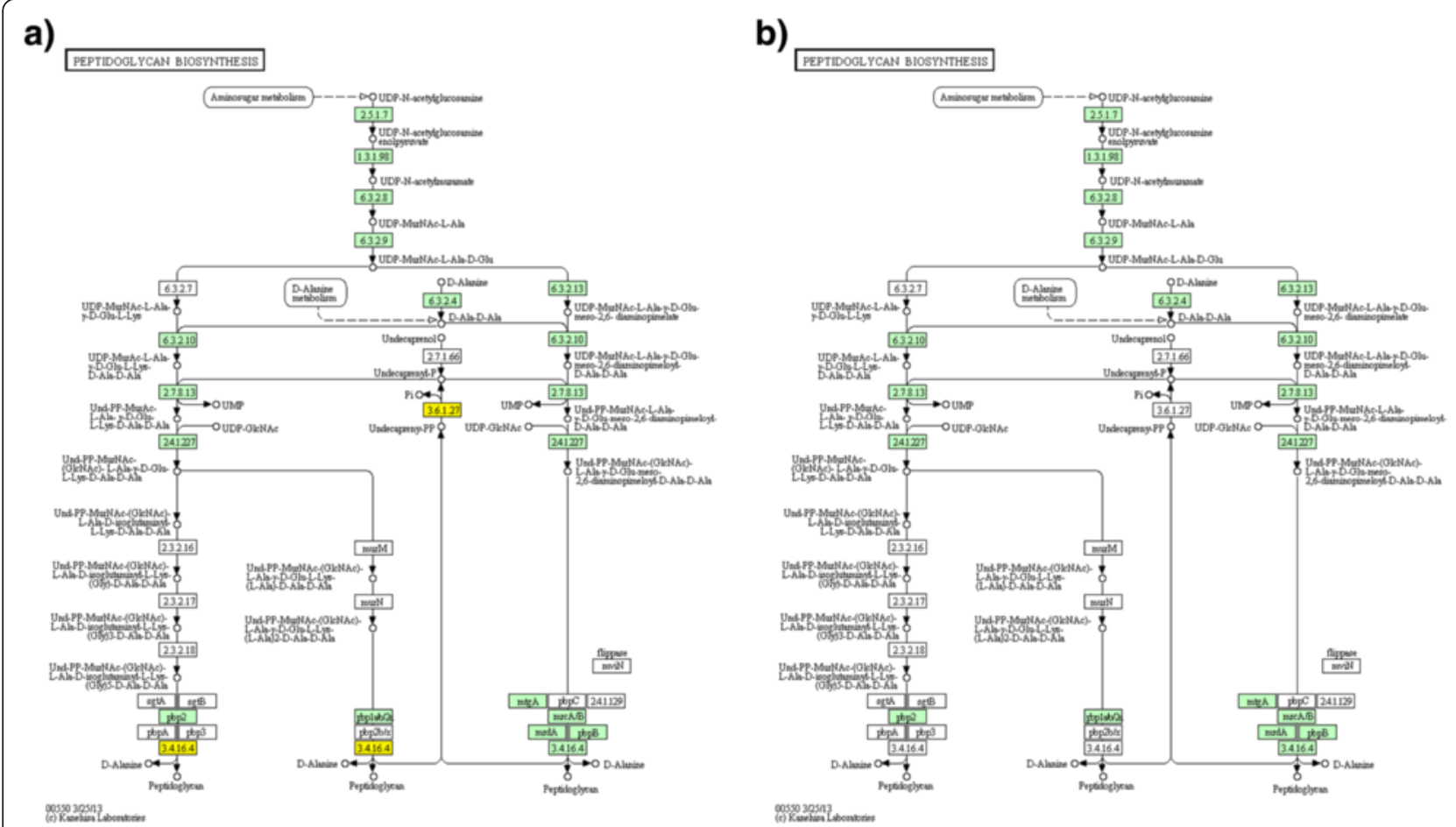

Figure 8 Peptidoglycan biosynthesis pathway in Prevotella intermedia ZT and the pan-genome of the genus Prevotella. a. The pangenome peptidoglycan biosynthesis pathway. The core genes are marked with green and the dispensable/specific genes with yellow. $\mathbf{b}$. The Prevotella intermedia ZT peptidoglycan biosynthesis pathway. The uncolored genes do not exist in the genome; They are shown in the graph to make the pathway explicit and integrated.

from empyema were sequenced. We isolated the strain Prevotella intermedia ZT from the infected root canal of a Chinese patient with chronic apical abscess and compared the genome content of this strain with other Prevotella taxa.

Our study confirmed that Prevotella intermedia ZT possessed a range of virulence factors involved in adhesion, proteolysis, iron uptake etc. For every aspect of pathogenicity, the involved genes have a relatively conserved part and a strain-specific part. The level of conservation varies with the aspect of pathogenicity, which indicates that the different strains of Prevotella intermedia inherited a basic package of genes enabling it to adapt to different niches and cause diseases, and later the package diverged under different conditions and the genes of different functions evolved at different rates. As far as this study shows, strain specificity is greater within genes involves in adhesion and the product and secretion of bacteriocin. It shows that, instead of exploiting the resources from the host, the most important variables of adaptation and pathogenicity are adhesion to the host tissue and/or surrounding microbes and competing with surrounding microbes. Besides, the activity of recombination shows that Prevotella intermedia may gain pathogenic genes through horizontal gene transfer.
Considering the similarity of the living environment and basis gene contents of the genus Prevotella, we analyze other Prevotella taxa to get a better view of what aspect of adaptation and pathogenicity matters most for the survival of Prevotella.

Our study shows that Prevotella taxa have very dynamic genomes with a part of relative high conserved contents. While comparing different taxa across species, we also find the existence of a conserved part. The genus Prevotella has a set of core genes involved in almost every COG class except "intracellular trafficking, secretion and vesicular transport" and "cell motility". The genomes also share an $\sim 49 \mathrm{~kb}$ conserved region involved in ribosome biosynthesis and translation. The pathways involved in carbohydrate metabolism and cell wall component synthesis are relatively conserved among the genus Prevotella. The anaerobic respiration product of genus Prevotella is neither lactate nor ethanol but potentially noxious chemicals such as formate. On the other hand, Prevotella taxa have very dynamic genomes. From the mathematics model, we draw the conclusion that the genus Prevotella have highly dynamic genomes that vary greatly in sequences and gene contents from species to species: On average any two genomes of different species share only half of the genes. The strain-specific genes 
make a large part of each COG cluster in the pan genome, which allows the taxa of the genus Prevotella to adapt to various niches and to show various pathogenicities. The genus Prevotella display great difference in amino acid biosynthesis. Rumen-isolated species Prevotella ruminicola maintains the ability to synthesize all the amino acid while other oral taxa more or less lost some genes to convert other metabolites to amino acids. The gene loss in Prevotella intermedia is most severe.

This result shows that Prevotella taxa which parasitize in oral cavity have enough nitrogen sources so that they gradually lost gene of amino acid synthesis. However, the function of cell wall synthesis remains intact under a highly dynamic context, which accords with our previous hypothesis that adhesion is one of the main drives of the evolution of Prevotella intermedia.

With a highly dynamic genome which enables fast adaptation, metabolic pathway that product potentially noxious products and a wide range of virulence factors, Prevotella intermedia are well equipped for survival in different niches and pathogenicity. Our study shows that instead of exploiting the resources from the host, adhesion and the competition and horizontal gene transfer with the surrounding microbes may be the main drives of the evolution of Prevotella intermedia.

However, since the core content of the Prevotella intermedia has already provide the basic function for each process of pathogenicity, the variation in pathogenic genes is not very significant. Actually it leaves a question whether the variation causes or is caused by adaptation to different niches. Limited by the data resources, we cannot fully answer and validate this question, but we assumed that the almost full function of core content can explain why Prevotella intermedia is able to survive in various niches.

We use a new method to evaluate the variation of genome content. Venn diagram is a common method to visualize the differences in the gene contents. It is explicit and easy to understand but troublesome when more than 5 genomes are analyzed due to the limited expression of flat graph. Here we make use of a mathematics model provided by PGAP to summarize and quantify the tendency of the gene content variation between different genomes. The mathematics model can be served as a supplementary for Venn diagram.

\section{Method}

\section{The collection of clinical samples}

\section{Selection of patient}

The sample was collected from a primary periapical periodontitis patient (male, 34 years old) who had sought treatment for endodontic treatment of his mandibular right canine (tooth 43) diagnosed with apical periodontitis. Biting pain, percussion pain and tenderness to palpation were presented. There was a sinus tract over the buccal mucosa of tooth 44. Gutta-percha tracing of the sinus tract indicated the lesion was originated from the apical region of tooth 43 .

The patients had no systematic diseases and had received no previous pulp and periodontal therapy and no antibiotics usage within 3 months before sample collection. The tooth harboring pulpal necrosis was sampled and the tooth was intact enough to allow adequate isolation from saliva.

We obtained the written informed consent from the patient, and the study was approved by the Ethic Committee of Shanghai 9th People's Hospital affiliated to Shanghai Jiao Tong University, School of Medicine, China. The age, gender, general conditions, prior oral treatment and preoperative radiograph were recorded.

\section{Sample collection}

The microbiological samples were collected from the infected root canals as described in previous studies [16,71]. After isolated from the oral cavity by a disinfected rubber dam, the tooth was disinfected with $3 \%$ hydrogen peroxide followed by $2.5 \%$ sodium hypochlorite, which was inactivated with $5 \%$ sodium thiosulfate to avoid interference with bacteriological sampling. The enamel was removed with sterile high-speed fissure bur and the operation area was disinfected again. The initial entry to pulp space was made by sterile burs under manual irrigation with sterile $0.9 \%$ saline solution. The patency of the root canal was established by a sterile $15 \mathrm{~K}$-type file without any usage of chemical solvents. Consecutively autoclave-sterilized dry paper points were inserted in the full length of the canal using the preoperative radiograph so that the fluid inside the root canal was absorbed. If the canal was dry, a little sterile $0.9 \%$ saline would be syringed into it first. The paper points per root canal were pooled into a $1.5 \mathrm{ml}$ centrifuge tube containing $1 \mathrm{ml}$ sterile pre-reduced PBS (Phosphate Buffered Saline) and transported to Shanghai Key Laboratory of Stomatology, China for culture within 20 min.

\section{Culture isolation}

The clinical transport fluid mentioned above was diluted $100 \mathrm{X}$ and $50 \mu \mathrm{l}$ dilution was inoculated into anaerobic blood agar CDC-based selective medium for black pigmented bacteria [72-74]. The content of the medium was 48 g CDC powder (Hangzhou Tianhe Microorganism Reagent Co., Ltd), $50 \mathrm{ml}$ sheep blood, $1 \mathrm{ml}$ Vitamin K1, $0.1 \mathrm{~g}$ kanamycin (Sangon Biotech (Shanghai) Co., Ltd) and $7.5 \mathrm{mg}$ vancomycin (Japan, Eli Lily Japan KK) dissolved in $1 \mathrm{~L}$ distilled water. The plates were cultured in an anaerobic incubator (UK, Ruskinn) in an atmosphere of $85 \%$ $\mathrm{N}_{2}, 10 \% \mathrm{CO}_{2}, 5 \% \mathrm{H}_{2}$ at $37^{\circ} \mathrm{C}$ for 7 days. 95 black colonies appeared in 3 of the 13 samples and were all isolated for 
further purification by subculture. The pure colonies were picked into eppendorf tubes and stored at $-20^{\circ} \mathrm{C}$.

\section{PCR testing}

Genome DNA of the 95 purified clones and standard strain Prevotella intermedia ATCC25611 supplied by Shanghai Key Laboratory of Stomatology, China were extracted with TIANamp Bacteria DNA Kit (Tiangen Biotech Co., LTD, Beijing, China) according to the manual. The DNA concentration of the samples is measured with spectrophotometer (Thermo Fisher Scientific Co., Ltd, Massachusetts, USA). The samples were diluted to $5 \mathrm{ng} / \mu \mathrm{l}$ and amplified with Prevotella intermedia specific PCR primers with the expected product length of $576 \mathrm{bp}$ : the forward primer: 5 '-TTTGTTGGGGAGTAAAGCG GG-3'; the reverse primer: 5'-TCAACATCTCTGTATC CTGCGT-3' (Sangon Biotech Co., Ltd, Shanghai, China). $1 \mu \mathrm{l}$ DNA template was added to the PCR mixture containing $12.5 \mu$ Premix Ex Taq vention (Biotechnology Co., LTD, Dalian, China) and $0.5 \mu \mathrm{l}$ of each primer and $10.5 \mu \mathrm{l}$ sterilized ultrapure water. The PCR was carried out in a thermal cycle (Applied Biosystems, California, USA) under the program as follows: $94^{\circ} \mathrm{C}$ for $10 \mathrm{~min}, 35$ cycles at $94^{\circ} \mathrm{C}$ for $1 \mathrm{~min}, 60^{\circ} \mathrm{C}$ for $30 \mathrm{~s}, 72^{\circ} \mathrm{C}$ for $30 \mathrm{~s}$, and a final $72^{\circ} \mathrm{C}$ for $7 \mathrm{~min}$. PCR products were analyzed with $1 \%$ agarose gel electrophorisis. The agarose gel was stained by ethidium bromide, and the image of the gel was captured under UV light by CCD camera. The sample that yielded the predicted amplicon of $576 \mathrm{bp}$ were predicted to belong to Prevotella intermedia species. They were selected and sent to Shanghai genomePilot Technology, Inc for wholelength $16 \mathrm{~s}$ rDNA sequencing. The DNA sample were applified with general $16 \mathrm{~s}$ DNA primer Forward 5'-AG AGTTTGATCCTGGCTCAG-3', Reverse 5'-ACGGCT ACCTTGTTACGACTT-3'. The Product is sequenced and compared against NCBI database with nBLAST tool. The result confirmed that the strains belonged to Prevotella intermedia species

\section{Genome sequencing, assembly and annotation}

The genomic DNA was sequenced by Roche 454 GS FLX system according to the standard protocol provided by the manufacturer and using the default parameter. 309,368 reads totaling $123,745,995$ nucleotides with an average length of $400 \mathrm{bp}$ per read were generated, providing approximately $65 \mathrm{X}$ of genome coverage. The raw data was trimmed using SeqClean and Lucy (1.20p version) to eliminate joint sequences generated during library building and sequencing. 871 reads shorter than $50 \mathrm{bp}$ were also left out. 167 contaminated reads from other species were detected by blasting against the NCBI NT Database and omitted from further analysis. 308,007 reads totaling 123,017,649 bp remained, $99.79 \%$ of which were assembled by Newbler 2.5.3 into 89 contigs amounting to $2,758,856 \mathrm{bp}$ and 320 singletons amounting to $53,951 \mathrm{bp}$.

ORF prediction was performed using GLIMMER 3.0. The ORF sequences were in silico translated and search against Non-redundant protein sequences database with blastp with the threshold of e $<1 \mathrm{e}-3$ and identity $>40 \%$. Only the best hits were used for gene function prediction. tRNA, tmRNA, rRNA were predicted by tRNAscan-SE (http://lowelab.ucsc.edu/tRNAscan-SE/), ARAGORN and RNAmmer1.2 respectively.

Other genomes of the genus Prevotella were downloaded from NCBI ftp (ftp://ftp.ncbi.nih.gov/genomes/).

The predicted protein sequences were classified with on-line COG engine WebMGA [75], which aligned the query sequences to the COG database updated on Feb 2nd 2011 with rpsblast 2.2.15 [76].

The protein sequences were mapped to KEGG [59] pathway using the online server KAAS [77] (http://www. genome.jp/tools/kaas/).

\section{Genomic sequence comparison}

The genome comparison was carried out using MUMmer 3.23. The analysis was performed according to the online manual (http://mummer.sourceforge.net/manual/) and the result was visualized by mapview.

Gene comparison was carried out using Blastn, with the threshold of e-value $=10^{-10}$, coverage $=80 \%$, identity $=60 \%$.

\section{Pan-genome analysis with PGAP}

Pan-Genomes Analysis Pipeline (PGAP) is a perl-script pipeline that consists of orthologous gene clustering, pan-genome analysis, SNP in CDS calling, evolutionary analysis and COG distribution.

The orthologous gene clustering was performed with The GeneFamily Method and ran as the following: The genes were first aligned using BLASTP with the following threshold: e-value of $1 \mathrm{e}-10$ and the score of 40 . Only gene pairs of Bidirectional Best Hits results (the identity greater than 50 and coverage greater than 0.5 from both sides) were clustered together. The other analysis was performed using the default parameter provided by the pipeline.

\section{KEGG pathway analysis}

The predicted ORFs were mapped to the KEGG pathway by KEGG Automatic Annotation Server (KAAS) [77]. The Genes data set was the default "for prokaryotes" and the assignment method was bi-directional best hit.

\section{Prediction of protease}

We predicted genes for proteases by two methods: we 1) searched among the annotation of ORFs and 2) searched all the predicted ORFs against the MEROPS release 8.9 
Protease Database. The 2 sets of results were then merged. The genes predicted to be protease by MEROPS but annotated to be enzymes other than protease with a high score and identity by BLAST were manually removed.

We further aligned all the predicted protease genes against the PSORTb [43] for the cellular location.

\section{Phylogenetics of the genus Prevotella}

In order to infer the phylogenetic relationships of the Prevotella intermedia ZT strain and the other 35 Prevotella taxa (Additional file 3), we first downloaded the genome data from NCBI ftp (ftp://ftp.ncbi.nih.gov/). Then the putative orthologous gene sets were generated through the following process: we first compared the all the 2067 predicted translated ORF sequences of Prevotella intermedia ZT strain against other taxa of the genus Prevotella by BLASTP method with the cutoff e-value of 0.1. 717 genes queries that have at least one hit in every species were selected for further analysis. Every query and its best representations of each species were viewed as a set of putative homologous genes. From these gene sets we manually screened for those that were 1) annotated to have the same biological function and 2) the housekeeping genes which are not likely to be influenced by horizontal gene transfer. 80 gene sets were picked and aligned and by ClustalW2.1 [78] with default parameter. The alignment files were clipped and concatenated into a set of 34581-nt multi-gene sequence with the help of BioEdit7.0.

The phylogenetic trees were built with Maximum Likelihood and Neighbor joining method in the Molecular Evolutionary Genetics Analysis (MEGA5.10) [79]. For the ML method, Jones-Taylor-Thornton (JTT) model was used and the result was tested by bootstrap method with the bootstrap replications of 1,000 . The rates among sites were set as uniform and all the gap and missing data were deleted. The NJ tree was generated using Poisson method and also tested by 1000replication bootstrap.

\section{Availability of supporting data}

The genome sequence data of Prevotella intermedia ZT (BioProject ID: PRJNA208776; accession: ATMK 00000000; BioSample SAMN02212661) is available at NCBI http://www.ncbi.nlm.nih.gov/bioproject/208776.

\section{Additional files}

Additional file 1: The clustered strain specific genes in Prevotella intermedia.

Additional file 2: The visualized genomic alignments of Prevotella taxa.

Additional file 3: The Prevotella taxa used in genome comparison and phylogenetics analysis.
Additional file 4: The housekeeping genes used in phylogenetic tree building.

\section{Abbreviations}

CDS: Coding DNA Sequence; ORF: Open reading frame;

LPS: Lipopolysaccharide; Pi: Prevotella intermedia.

\section{Competing interests}

The authors declare that they have no competing interests.

\section{Authors' contributions}

YR and LS carried out the data analysis, participated in the sequencing and drafted the manuscript. $Z Q$ and $Y Z$ isolated the strain and extracted the genome DNA. JY, LG, ZT participated in the clinical sample collection. LH ZC and JJ gave advice on the data analysis and helped to draft the manuscript. SQ and ZT designed the study. All authors read and approved the final manuscript.

\section{Acknowledgements}

We thank all the participants in this study. It was supported by grants from the 863 Program (2012AA02A515), the 973 Program (2010CB529600), the National Key Technology R\&D Program (2012BAI01B09), the National Nature Science Foundation of China (81121001, 81273596,30900799, 30972823), the Shanghai Municipal Commission of Science and Technology Program (11DZ1950300, 09DJ1400601), the Shanghai Jiaotong University Med-X Fund (YG2010MS61), Public Science and Technology Research Funds (201210056), the Shanghai Jiaotong University Interdisciplinary Research fund, the Shanghai Leading Academic Discipline Project (B205), Shanghai Public Health Leading Academic Discipline Project(12GWZX0801) and the Public Health Key Disciplines in Shanghai-Health Microbiology (No.12GWZX0801).

\section{Author details}

${ }^{1}$ Bio-X Institutes, Key Laboratory for the Genetics of Developmental and Neuropsychiatric Disorders(Ministry of Education, Shanghai Jiao Tong University, 1954 Huashang Road, Shanghai 200030, China. ${ }^{2}$ Shanghai Institutes of Pilot Genomics and Human Health, Shanghai 200030, China. ${ }^{3}$ The Fourth Hospital of Jinan City; Taishan Medical College, Jinan 250031, China. ${ }^{4}$ Center for Reproductive Medicine, Shandong Provincial Hospital, Shandong University, Jinan, Shandong, China. ${ }^{5}$ Department of Endodontics, 9th People's Hospital, Shanghai JiaoTong University, School of Medicine, Shanghai Key Laboratory of Stomatology, Shanghai 200011, China.

Received: 15 November 2014 Accepted: 22 January 2015

Published online: 25 February 2015

\section{References}

1. Shah HN, Collins DM. NOTES: Prevotella, a new genus to include bacteroides melaninogenicus and related species formerly classified in the genus bacteroides. Int J Systematic. 1990;40(2):205-8.

2. Eiring $P$, Waller $K$, Widmann $A$, Werner H. Fibronectin and laminin binding of urogenital and oral prevotella species. Zentralbl Bakteriol. 1998;288(3):361-72.

3. Hayashi H, Shibata K, Sakamoto M, Tomita S, Benno Y. Prevotella copri sp. nov. and Prevotella stercorea sp. nov., isolated from human faeces. Int J Syst Evol Microbiol. 2007;57(Pt 5):941-6.

4. Gomes BP, Lilley JD, Drucker DB. Associations of endodontic symptoms and signs with particular combinations of specific bacteria. Int Endod J. 1996;29(2):69-75.

5. Gomes BP, Drucker DB, Lilley JD. Associations of specific bacteria with some endodontic signs and symptoms. Int Endod J. 1994;27(6):291-8.

6. Ulrich M, Beer I, Braitmaier P, Dierkes M, Kummer F, Krismer B, et al. Relative contribution of Prevotella intermedia and Pseudomonas aeruginosa to lung pathology in airways of patients with cystic fibrosis. Thorax. 2010;65(11):978-84.

7. Nadkarni MA, Browne GV, Chhour K, Byun R, Nguyen K, Chapple CC, et al. Pattern of distribution of Prevotella species/phylotypes associated with healthy gingiva and periodontal disease. Eur J Clin Microbiol Infect Dis. 2012;31(11):2989-99. 
8. Fujii R, Saito Y, Tokura Y, Nakagawa Kl, Okuda K, Ishihara K. Characterization of bacterial flora in persistent apical periodontitis lesions. vol. 24. Blackwell Publishing Ltd; 2009. p. 502-5.

9. Field TR, Sibley CD, Parkins MD, Rabin HR, Surette MG. The genus Prevotella in cystic fibrosis airways. Anaerobe. 2010;16(4):337-44.

10. Maeda N, Okamoto M, Kondo K, Ishikawa H, Osada R, Tsurumoto A, et al. Incidence of Prevotella intermedia and Prevotella nigrescens in periodontal health and disease. Microbiol Immunol. 1998:42(9):583-9.

11. Mombelli A, Schmid B, Rutar A, Lang NP. Persistence patterns of Porphyromonas gingivalis, Prevotella intermedia/nigrescens, and Actinobacillus actinomyetemcomitans after mechanical therapy of periodontal disease. J Periodontol. 2000;71(1):14-21.

12. Ashimoto A, Chen C, Bakker I, Slots J. Polymerase chain reaction detection of 8 putative periodontal pathogens in subgingival plaque of gingivitis and advanced periodontitis lesions. Oral Microbiol Immunol. 1996;11(4):266-73.

13. Jacinto RC, Gomes BP, Ferraz CC, Zaia AA, Filho FJ. Microbiological analysis of infected root canals from symptomatic and asymptomatic teeth with periapical periodontitis and the antimicrobial susceptibility of some isolated anaerobic bacteria. Oral Microbiol Immunol. 2003;18(5):285-92.

14. Bolivar I, Whiteson K, Stadelmann B, Baratti-Mayer D, Gizard Y, Mombelli A, et al. Bacterial diversity in oral samples of children in Niger with acute noma, acute necrotizing gingivitis, and healthy controls. PLoS Negl Trop Dis. 2012;6(3):e1556.

15. Jr Falkler WA, Enwonwu CO, Idigbe EO. Microbiological understandings and mysteries of noma (cancrum oris). Oral Dis. 1999:5(2):150-5.

16. Gomes BP, Jacinto RC, Pinheiro ET, Sousa EL, Zaia AA, Ferraz CC, et al. Porphyromonas gingivalis, Porphyromonas endodontalis, Prevotella intermedia and Prevotella nigrescens in endodontic lesions detected by culture and by PCR. Oral Microbiol Immunol. 2005;20(4):211-5.

17. Gomes BP, Pinheiro ET, Gade-Neto CR, Sousa EL, Ferraz CC, Zaia AA, et al. Microbiological examination of infected dental root canals. Oral Microbiol Immunol. 2004;19(2):71-6.

18. Brook I, Frazier EH. IMmune response to fusobacterium nucleatum and prevotella intermedia in the sputum of patients with acute exacerbation of chronic bronchitis*. Chest J. 2003;124(3):832-3.

19. Hamada S, Amano A, Kimura S, Nakagawa I, Kawabata S, Morisaki I. The importance of fimbriae in the virulence and ecology of some oral bacteria. Oral Microbiol Immunol. 1998;13(3):129-38.

20. Sanchez CJAS. The Pneumococcal serine-rich repeat protein is an intraspecies bacterial adhesin that promotes bacterial aggregation in vivo and in Biofilms. PLoS Pathog. 2010;6(8):e1001044.

21. Piessens V, De Vliegher S, Verbist B, Braem G, Van Nuffel A, De Vuyst L, et al. Intra-species diversity and epidemiology varies among coagulase-negative Staphylococcus species causing bovine intramammary infections. Vet Microbiol. 2012:155(1):62-71.

22. Lan R, Reeves PR. Intraspecies variation in bacterial genomes: the need for a species genome concept. Trends Microbiol. 2000;8(9):396-401.

23. Iyer D, Anaya-Bergman C, Jones K, Yanamandra S, Sengupta D, Miyazaki H, et al. AdpC is a Prevotella intermedia 17 leucine-rich repeat internalin-like protein. Infect Immun. 2010;78(6):2385-96.

24. Yu F, Iyer D, Anaya C, Lewis JP. Identification and characterization of a cell surface protein of Prevotella intermedia 17 with broad-spectrum binding activity for extracellular matrix proteins. Proteomics. 2006;6(22):6023-32.

25. He J. Characterization of adhesins and other virulence factors involved in the interaction of prevotella intermedia with human epithelial cells. Hangzhou: Zhejiang University; 2010.

26. Holt SC, Kesavalu L, Walker S, Genco CA. Virulence factors of Porphyromonas gingivalis. Periodontol 2000. 1999;20:168-238.

27. Turk B. Targeting proteases: successes, failures and future prospects. Nat Rev Drug Discov. 2006:5(9):785-99.

28. Lopez-Otin C, Hunter $T$. The regulatory crosstalk between kinases and proteases in cancer. Nat Rev Cancer. 2010;10(4):278-92.

29. Jansen HJ, Grenier D, Van der Hoeven JS. Characterization of immunoglobulin G-degrading proteases of Prevotella intermedia and Prevotella nigrescens. Oral Microbiol Immunol. 1995;10(3):138-45.

30. Deschner J, Singhal A, Long P, Liu CC, Piesco N, Agarwal S. Cleavage of CD14 and LBP by a protease from Prevotella intermedia. Arch Microbiol. 2003:179(6):430-6.

31. Jansen HJ, van der Hoeven JS, Walji S, Goertz JH, Bakkeren JA. The importance of immunoglobulin-breakdown supporting the growth of bacteria in oral abscesses. J Clin Periodontol. 1996;23(8):717-23.
32. Leung KP, Subramaniam PS, Okamoto M, Fukushima $\mathrm{H}$, Lai $\mathrm{CH}$. The binding and utilization of hemoglobin by Prevotella intermedia. Fems Microbiol Lett. 1998;162(2):227-33.

33. Leung KP, Fukushima H, Nesbitt WE, Clark WB. Prevotella intermedia fimbriae mediate hemagglutination. Oral Microbiol Immunol. 1996;11(1):42-50.

34. Okamoto M, Maeda N, Kondo K, Leung KP. Hemolytic and hemagglutinating activities of Prevotella intermedia and Prevotella nigrescens. Fems Microbiol Lett. 1999;178(2):299-304.

35. Nakayama K, Ratnayake DB, Tsukuba T, Kadowaki T, Yamamoto K, Fujimura S. Haemoglobin receptor protein is intragenically encoded by the cysteine proteinase-encoding genes and the haemagglutinin-encoding gene of Porphyromonas gingivalis. Mol Microbiol. 1998;27(1):51-61.

36. Silva TA, Rodrigues PH, Ribeiro RN, Noronha FS, Farias LM, Carvalho MA. Hemolytic activity of Prevotella intermedia and Prevotella nigrescens strains: influence of abiotic factors in solid and liquid assays. Res Microbiol. 2003;154(1):29-35.

37. Beem JE, Nesbitt WE, Leung KP. Identification of hemolytic activity in Prevotella intermedia. Oral Microbiol Immunol. 1998;13(2):97-105.

38. Takada K, Fukatsu A, Otake S, Hirasawa M. Isolation and characterization of hemolysin activated by reductant from Prevotella intermedia. FEMS Immunol Med Microbiol. 2003;35(1):43-7.

39. Guan SM, Nagata H, Shizukuishi S, Wu JZ. Degradation of human hemoglobin by Prevotella intermedia. Anaerobe. 2006;12(5-6):279-82.

40. Byrne DP, Wawrzonek K, Jaworska A, Birss AJ, Potempa J, Smalley JW. Role of the cysteine protease interpain A of Prevotella intermedia in breakdown and release of haem from haemoglobin. Biochem J. 2010;425(1):257-64.

41. Smalley JW, Silver J, Birss AJ, Withnall R, Titler PJ. The haem pigment of the oral anaerobes Prevotella nigrescens and Prevotella intermedia is composed of iron(III) protoporphyrin IX in the monomeric form. Microbiology. 2003;149(Pt 7):1711-8.

42. Potempa M, Potempa J, Kantyka T, Nguyen KA, Wawrzonek K, Manandhar SP, et al. Interpain A, a cysteine proteinase from Prevotella intermedia, inhibits complement by degrading complement factor C3, vol. 5. United States: PLoS pathogens; 2009

43. Yu NY, Wagner JR, Laird MR, Melli G, Rey S, Lo R, et al. PSORTb 3.0: improved protein subcellular localization prediction with refined localization subcategories and predictive capabilities for all prokaryotes. Bioinformatics. 2010;26(13):1608-15

44. Shoji M, Shibata Y, Shiroza T, Yukitake H, Peng B, Chen Y, et al. Characterization of hemin-binding protein 35 (HBP35) in Porphyromonas gingivalis: its cellular distribution, thioredoxin activity and role in heme utilization. BMC Microbiol. 2010;10(1):152.

45. Dashper SG, Hendtlass A, Slakeski N, Jackson C, Cross KJ, Brownfield L, et al. Characterization of a novel outer membrane hemin-binding protein of Porphyromonas gingivalis. J Bacteriol. 2000;182(22):6456-62.

46. Xu X, Kolodrubetz D. Construction and analysis of hemin binding protein mutants in the oral pathogen Treponema denticola. Res Microbiol. 2002;153(9):569-77.

47. Shibata Y, Miwa Y, Hirai K, Fujimura S. Purification and partial characterization of a dipeptidyl peptidase from Prevotella intermedia. Oral Microbiol Immunol. 2003;18(3):196-8.

48. Chen X. Biochemical properties of recombinant prolyl dipeptidases DPP-IV and DPP8. Adv Exp Med Biol. 2006;575:27-32.

49. Engel M, Hoffmann T, Wagner L, Wermann M, Heiser U, Kiefersauer R, et al. The crystal structure of dipeptidyl peptidase IV (CD26) reveals its functional regulation and enzymatic mechanism. Proc Natl Acad Sci U S A. 2003;100(9):5063-8.

50. Kahne T, Lendeckel U, Wrenger S, Neubert K, Ansorge S, Reinhold D. Dipeptidyl peptidase IV: a cell surface peptidase involved in regulating T cell growth (review). Int J Mol Med. 1999;4(1):3-15

51. Pro B, Dang NH. CD26/dipeptidyl peptidase IV and its role in cancer. Histol Histopathol. 2004;19(4):1345-51.

52. Kelly T. Fibroblast activation protein-a and dipeptidyl peptidase IV (CD26): Cell-surface proteases that activate cell signaling and are potential targets for cancer therapy. Drug Resist Update. 2005;8(1-2):51-8.

53. Gazi MI, Cox SW, Clark DT, Eley BM. Comparison of host tissue and bacterial dipeptidyl peptidases in human gingival crevicular fluid by analytical isoelectric focusing. Arch Oral Biol. 1995;40(8):731-6.

54. Fernandez RC, Weiss AA. Cloning and sequencing of a Bordetella pertussis serum resistance locus. Infect Immun. 1994;62(11):4727-38. 
55. Tatusov RL, Koonin EV, Lipman DJ. A genomic perspective on protein families. Science. 1997;278(5338):631-7.

56. Medini D, Donati C, Tettelin H, Masignani V, Rappuoli R. The microbial pan-genome. Curr Opin Genet Dev. 2005;15(6):589-94.

57. Persson R, Hitti J, Verhelst R, Vaneechoutte M, Persson R, Hirschi R, et al. The vaginal microflora in relation to gingivitis. BMC Infect Dis. 2009;9:6.

58. Egwari L, Rotimi VO, Abudu OO, Coker AO. A study of the anaerobic bacterial flora of the female genital tract in health and disease. Cent Afr J Med. 1995:41(12):391-7.

59. Kanehisa M, Goto S. KEGG: Kyoto Encyclopedia of Genes and Genomes. Nucleic Acids Res. 2000;28(1):27-30.

60. Takahashi N, Yamada T. Pathways for amino acid metabolism by Prevotella intermedia and Prevotella nigrescens, vol. 15. United States: Oral Microbiology and Immunology; 2000. p. 96-102.

61. Takahashi N, Yamada T. Glucose metabolism by Prevotella intermedia and Prevotella nigrescens, vol. 15. United States: Molecular Oral Microbiology; 2000. p. 188-95.

62. Iki K, Kawahara K, Sawamura S, Arakaki R, Sakuta T, Sugiyama A, et al. A novel component different from endotoxin extracted from Prevotella intermedia ATCC 25611 activates lymphoid cells from C3H/HeJ mice and gingival fibroblasts from humans. Infect Immun. 1997:65(11):4531-8.

63. Sugiyama A, Uehara A, Iki K, Matsushita K, Nakamura R, Ogawa T, et al. Activation of human gingival epithelial cells by cell-surface components of black-pigmented bacteria: augmentation of production of interleukin-8, granulocyte colony-stimulating factor and granulocyte-macrophage colony-stimulating factor and expression of intercellular adhesion molecule 1. J Med Microbiol. 2002;51(1):27-33.

64. Nagaoka S, Tokuda M, Sakuta T, Taketoshi Y, Tamura M, Takada H, et al. Interleukin-8 gene expression by human dental pulp fibroblast in cultures stimulated with Prevotella intermedia lipopolysaccharide. J Endod. 1996;22(1):9-12.

65. Kirikae T, Nitta T, Kirikae F, Suda Y, Kusumoto S, Qureshi N, et al, Lipopolysaccharides (LPS) of oral black-pigmented bacteria induce tumor necrosis factor production by LPS-refractory $\mathrm{C} 3 \mathrm{H} / \mathrm{HeJ}$ macrophages in a way different from that of Salmonella LPS. Infect Immun. 1999;67(4):1736-42.

66. Tamura M, Tokuda M, Nagaoka S, Takada H. Lipopolysaccharides of Bacteroides intermedius (Prevotella intermedia) and Bacteroides (Porphyromonas) gingivalis induce interleukin-8 gene expression in human gingival fibroblast cultures. Infect Immun. 1992;60(11):4932-7.

67. Kim SJ, Ha MS, Choi EY, Choi Jl, Choi IS. Prevotella intermedia lipopolysaccharide stimulates release of nitric oxide by inducing expression of inducible nitric oxide synthase. J Periodontal Res. 2004;39(6):424-31.

68. Kim SJ, Choi EY, Kim EG, Shin SH, Lee JY, Choi Jl, et al. Prevotella intermedia lipopolysaccharide stimulates release of tumor necrosis factor-alpha through mitogen-activated protein kinase signaling pathways in monocyte-derived macrophages. FEMS Immunol Med Microbiol. 2007:51(2):407-13.

69. Tokuda M, Sakuta T, Fushuku A, Torii M, Nagaoka S. Regulation of interleukin-6 expression in human dental pulp cell cultures stimulated with Prevotella intermedia lipopolysaccharide. J Endod. 2001;27(4):273-7.

70. Page RC. The role of inflammatory mediators in the pathogenesis of periodontal disease. J Periodontal Res. 1991;26(3 Pt 2):230-42.

71. Ng Y, Spratt D, Sriskantharajah S, Gulabivala K. Evaluation of protocols for field decontamination before bacterial sampling of root canals for contemporary microbiology techniques. J Endodont. 2003;29(5):317-20.

72. Holbrook WP, Ogston SA, Ross PW. A method for the isolation of Bacteroides melaninogenicus from the human mouth. J Med Microbiol. 1978;11(2):203-7.

73. Vianna ME, Horz HP, Gomes BPFA, Conrads G. Microarrays complement culture methods for identification of bacteria in endodontic infections. vol. 20. Munksgaard International Publishers; 2005. p. 253-8.

74. van Winkelhoff AJ, de Graaff J. Vancomycin as a selective agent for isolation of Bacteroides species. J Clin Microbiol. 1983;18(5):1282-4

75. Wu S, Zhu Z, Fu L, Niu B, Li W. WebMGA: a customizable web server for fast metagenomic sequence analysis. BMC Genomics. 2011;12:444

76. Altschul SF, Gish W, Miller W, Myers EW, Lipman DJ. Basic local alignment search tool. J Mol Biol. 1990;215(3):403-10.

77. Moriya Y, Itoh M, Okuda S, Yoshizawa AC, Kanehisa M. KAAS: an automatic genome annotation and pathway reconstruction server. Nucleic Acids Res. 2007:35(Web Server issue):W182-5.
78. Larkin MA, Blackshields G, Brown NP, Chenna R, McGettigan PA, McWilliam H, et al. Clustal W and Clustal $X$ version 2.0. Bioinformatics. 2007;23(21):2947-8.

79. Tamura K, Peterson D, Peterson N, Stecher G, Nei M, Kumar S. MEGA5: molecular evolutionary genetics analysis using maximum likelihood, evolutionary distance, and maximum parsimony methods. Mol Biol Evol. 2011;28(10):2731-9.

\section{Submit your next manuscript to BioMed Central and take full advantage of:}

- Convenient online submission

- Thorough peer review

- No space constraints or color figure charges

- Immediate publication on acceptance

- Inclusion in PubMed, CAS, Scopus and Google Scholar

- Research which is freely available for redistribution 